\title{
Canonical transformations in quantum mechanics
}

\author{
Maciej Błaszak, Ziemowit Domański \\ Faculty of Physics, Adam Mickiewicz University \\ Umultowska 85, 61-614 Poznań, Poland
}

\begin{abstract}
This paper presents the general theory of canonical transformations of coordinates in quantum mechanics. First, the theory is developed in the formalism of phase space quantum mechanics. It is shown that by transforming a star-product, when passing to a new coordinate system, observables and states transform as in classical mechanics, i.e., by composing them with a transformation of coordinates. Then the developed formalism of coordinate transformations is transferred to a standard formulation of quantum mechanics. In addition, the developed theory is illustrated on examples of particular classes of quantum canonical transformations.
\end{abstract}

Keywords: quantum mechanics, deformation quantization, canonical transformations, Moyal product, phase space

\section{Introduction}

In classical Hamiltonian mechanics transformations of phase space coordinates (especially canonical transformations of coordinates) are an important part of the theory. Since quantum mechanics arises from Hamiltonian mechanics it is natural to ask if it is possible to introduce the concept of canonical transformations of coordinates in quantum mechanics. The importance of this problem was evident to scientists from the early days of quantum theory. The development of the theory of canonical transformations of coordinates in quantum mechanics is mainly contributed to Jordan, London and Dirac back in 1925 [1-7] and it is still an area of intense research.

In the usual approach to canonical transformations in quantum mechanics one identifies canonical transformations with unitary operators defined on a Hilbert space. Such approach was used by Mario Moshinsky and his collaborators in a series of papers 8-12]. Also other researchers used such approach [13, 14]. Worth noting are also papers of Anderson [15, 16] where an extension of canonical transformations to non-unitary operators is presented. Nevertheless, after so many years of efforts, there is still lack of a general theory of coordinate transformations in quantum mechanics, including a satisfactory complete theory of canonical transformations. With the following paper we are trying to fill this gap, at least to some extend.

A unitary operator $\hat{U}$ associated with some canonical change of coordinates transforms vector states from one coordinate system to the other: $\phi^{\prime}=\hat{U} \phi$. Also observables, being operators on a Hilbert space of states, transform according to a prescription

$$
\hat{A}^{\prime}=\hat{U} \hat{A} \hat{U}^{-1}
$$

where $\hat{A}$ is an initial operator and $\hat{A}^{\prime}$ an operator after the change of coordinates. In particular, operators $\hat{q}, \hat{p}$ of position and momentum transform according to (1). Observe that the commutator of operators of position and momentum as well as their Hermitian property do not change after a unitary transformation. For this reason unitary operators are identified with canonical transformations.

Email addresses: blaszakm@amu.edu.pl (Maciej Błaszak), ziemowit@amu.edu.pl (Ziemowit Domański) 
To some unitary operators one can try to associate transformations defined on a classical phase space. It can be done using a Born's quantization rule. This rule formulated by Max Born states that to every classical observable $A(x, p)$ (real-valued function defined on a phase space) written in a Cartesian coordinate system one can associate an operator on a Hilbert space by substituting for $x$ and $p$ operators of position and momentum $\hat{q}, \hat{p}$ and symmetrically ordering them. If one now considers a transformation of phase space coordinates (possibly depending on an evolution parameter $t$ )

$$
T=(Q, P): \mathbb{R}^{2} \supset U \rightarrow W \subset \mathbb{R}^{2},
$$

then one can define operators

$$
\begin{aligned}
& \hat{Q}=Q(\hat{q}, \hat{p}, t), \\
& \hat{P}=P(\hat{q}, \hat{p}, t) .
\end{aligned}
$$

If $[\hat{Q}, \hat{P}]=i \hbar$ then the operators $\hat{Q}, \hat{P}$ can be thought of as operators of position and momentum associated to a new coordinate system, and $T$ is then called a quantum canonical transformation. In some cases the operator $\hat{Q}$ can be written in a position representation, i.e., as an operator of multiplication by a coordinate variable. In fact, there exist a measure $\mu$ and a unitary operator $\hat{U}_{T}: \mathcal{H} \rightarrow L^{2}(\sigma(\hat{Q}), \mu)$ such that

$$
\hat{U}_{T} \hat{Q} \hat{U}_{T}^{-1}=x^{\prime}
$$

where $\sigma(\hat{Q})$ is the spectrum of the operator $\hat{Q}$. The operator $\hat{U}_{T}$ is precisely the unitary operator corresponding to the quantum canonical transformation $T$.

At this point we arrive to a problem concerning the Born's quantization procedure. Namely, we could try to quantize a classical Hamiltonian system in some arbitrary canonical coordinate system using the Born's quantization rule. However, this way we would end up in non-equivalent quantum systems. As an example let us consider a classical harmonic oscillator which time evolution is governed by the Hamiltonian

$$
H(x, p)=\frac{1}{2}\left(p^{2}+\omega^{2} x^{2}\right)
$$

In accordance with the Born's quantization rule to $H$ corresponds the following operator

$$
\hat{H}=H(\hat{q}, \hat{p})=\frac{1}{2}\left(\hat{p}^{2}+\omega^{2} \hat{q}^{2}\right) .
$$

Performing a classical canonical transformation of coordinates $T:(\mathbb{R} \backslash\{0\}) \times \mathbb{R} \rightarrow(\mathbb{R} \backslash\{0\}) \times \mathbb{R}, T\left(x^{\prime}, p^{\prime}\right)=$ $(x, p)$ where

$$
x=\left\{\begin{array}{rl}
\sqrt{\left|2 x^{\prime}\right|}, & x^{\prime}>0 \\
-\sqrt{\left|2 x^{\prime}\right|}, & x^{\prime}<0
\end{array}, \quad p=p^{\prime} \sqrt{\left|2 x^{\prime}\right|},\right.
$$

with the inverse $T^{-1}$ in the form

$$
x^{\prime}=\left\{\begin{array}{rr}
\frac{1}{2} x^{2}, & x>0 \\
-\frac{1}{2} x^{2}, & x<0
\end{array}, \quad p^{\prime}=|x|^{-1} p,\right.
$$

the Hamiltonian $H$ transforms to the following function

$$
H^{\prime}\left(x^{\prime}, p^{\prime}\right)=H\left(T\left(x^{\prime}, p^{\prime}\right)\right)=\left|x^{\prime}\right| p^{\prime 2}+\omega^{2}\left|x^{\prime}\right| .
$$

Applying the Born's quantization rule to $H^{\prime}$ with symmetric ordering of canonical operators of position and momentum, $\hat{q}^{\prime}=x^{\prime}, \hat{p}^{\prime}=-i \hbar \partial_{x^{\prime}}$, results in the following operator

$$
\hat{H}^{\prime}=H^{\prime}\left(\hat{q}^{\prime}, \hat{p}^{\prime}\right)=\frac{1}{2}\left|\hat{q}^{\prime}\right| \hat{p}^{\prime 2}+\frac{1}{2} \hat{p}^{\prime 2}\left|\hat{q}^{\prime}\right|+\omega^{2}\left|\hat{q}^{\prime}\right| .
$$


Hamiltonians $\hat{H}$ and $\hat{H}^{\prime}$ are not unitarily equivalent - they describe different quantum systems. As we will show in this paper, the apparent inconsistency of the quantization can be solved by changing the ordering of operators $\hat{q}^{\prime}, \hat{p}^{\prime}$ in the transformed Hamiltonian $H^{\prime}$ in an appropriate way, which will be equivalent to the existence of a respective unitary operator related to the transformation.

In order to control the whole procedure we take a different route for developing a theory of transformations of coordinates in quantum mechanics, to that usually found in the literature. Namely, first we consider a quantum theory on a phase space, and then pass to the standard description of quantum mechanics. Such approach seems to be more natural as the concept of a change of coordinates can be introduced in a similar manner as in classical Hamiltonian theory.

In the usual approach to coordinate transformations in phase space quantum mechanics [17 19] one modifies an action of a transformation of coordinates on observables and states, from the simple composition of maps. This is done to receive a consistent theory. We present a slightly different, although equivalent, approach in which we transform not only observables and states but also a star-product. Such an idea the reader can also find in [20]. Then the action of a coordinate transformation on phase space functions can remain to be a simple composition of maps. Moreover, we present a systematic way of passing the developed theory of transformations to the standard description of quantum mechanics. Such approach of dealing with change of coordinates in quantum theory proves to be very effective and gives means to develop a general theory of transformations of coordinates in quantum mechanics which is still missing after almost 90 years of its development.

The paper is organized as follows. In Section 2 we review basic concepts of phase space quantum mechanics focusing on properties important in developing a transformation theory. Section 3 presents the general theory of canonical transformations of coordinates in phase space quantum mechanics. Section 4 contains the passage of the quantum transformation theory developed on the phase space to the ordinary quantum mechanics, using the results from previous section. Section 5 presents couple classes of transformations of coordinates illustrating the developed formalism. In Section 6 we give final remarks and conclusions.

\section{Phase space quantum mechanics}

\subsection{Preliminaries}

There exist many approaches to quantum mechanics. The one which seems to be the most suited to develop a theory of transformations of phase space coordinates is a phase space quantum mechanics also referred to as a deformation quantization (see [21, 22], and [23 25] for recent reviews). The review of phase space quantum mechanics which we will present is based on our earlier work 26].

The idea behind the phase space quantum mechanics relies on an appropriate deformation of a classical Hamiltonian system. Such approach to quantum theory uses a mathematical language very similar to that of a classical theory. This have many benefits. On the one hand it allows a straightforward introduction of concepts from classical mechanics into the quantum counterpart (for example, the concept of a transformation of phase space coordinates), on the other hand it gives in a natural way a classical Hamiltonian mechanics as a limiting case, hence allowing investigation of quantum systems in a classical limit.

The procedure of a deformation should be performed with respect to some parameter $\hbar$ (which is taken to be a Planck's constant) so that in the limit $\hbar \rightarrow 0$ the quantum theory should reduce to the classical theory.

The deformation of a classical Hamiltonian system can be fully given by deforming an algebraic structure of a classical Poisson algebra. This will then yield a deformation of a phase space (a Poisson manifold) to a noncommutative phase space (a noncommutative Poisson manifold), a deformation of classical states to quantum states and a deformation of classical observables to quantum observables.

First, let us deal with a deformation of a phase space. A Poisson manifold $(M, \mathcal{P})(\mathcal{P}$ being a Poisson tensor) is fully described by a Poisson algebra $\mathcal{A}_{C}=\left(C^{\infty}(M), \cdot,\{\cdot, \cdot\}\right)$ of smooth complex-valued functions on the phase space $M$, where $\cdot$ is a point-wise product of functions and $\{\cdot, \cdot\}$ is a Poisson bracket induced by a Poisson tensor $\mathcal{P}$. Hence by deforming $\mathcal{A}_{C}$ to some noncommutative algebra $\mathcal{A}_{Q}=\left(C^{\infty}(M), \star, \llbracket \cdot, \cdot \rrbracket\right)$, where $\star$ is some noncommutative associative product of functions being a deformation of a point-wise 
product, and $\llbracket \cdot, \cdot \rrbracket$ is a Lie bracket satisfying the Leibniz's rule and being a deformation of the Poisson bracket $\{\cdot, \cdot\}$, we can think of a quantum Poisson algebra $\mathcal{A}_{Q}$ as describing a noncommutative Poisson manifold.

The algebra $\mathcal{A}_{C}$ contains in particular a subset of classical observables, whereas $\mathcal{A}_{Q}$ contains a subset of quantum observables. Note that quantum observables are functions on the phase space $M$ similarly as in classical mechanics. Furthermore, classical observables are real-valued functions from $\mathcal{A}_{C}$, i.e., self-adjoint functions with respect to the complex-conjugation — an involution in the algebra $\mathcal{A}_{C}$. Quantum observables should also be self-adjoint functions with respect to an involution in the algebra $\mathcal{A}_{Q}$. However, in general the complex-conjugation do not need to be an involution in $\mathcal{A}_{Q}$. Thus in $\mathcal{A}_{Q}$ we have to introduce some involution which would be a deformation of the complex-conjugation [26]. As a consequence, quantum observables (selfadjoint functions with respect to the quantum involution) might be complex and $\hbar$-dependent.

There is a vast number of equivalent quantization schemes (see [26] for review of the subject) which yield a quantization equivalent to a standard approach to quantum mechanics but giving different orderings of position and momentum operators. From this diversity of quantization schemes the simplest one is a Moyal quantization. It follows from the fact that for the Moyal quantum algebra the involution is the complexconjugation as in the classical case. Thus in this case quantum observables, exactly like classical observables, can be chosen as real-valued functions. Further on we will deal only with that distinguished quantization. Such a choice is not a restriction as other quantization schemes known in the literature are gauge equivalent to the Moyal one (see [26] and Subsection [2.5)).

Throughout the paper we will use the following notation. The $i$-th components of vectors $x, p \in \mathbb{R}^{N}$ representing positions and momenta will be denoted by $x^{i}$ and $p_{i}$, i.e., $x=\left(x^{1}, \ldots, x^{N}\right)$ and $p=\left(p_{1}, \ldots, p_{N}\right)$. By $x p$ we will understand the scalar product of vectors $x$ and $p$, i.e., $x p=x^{i} p_{i}$. Often sequences of functions $\left(Q^{1}, \ldots, Q^{N}\right),\left(P_{1}, \ldots, P_{N}\right)$, derivatives $\left(\partial_{x^{1}}, \ldots, \partial_{x^{N}}\right),\left(\partial_{p_{1}}, \ldots, \partial_{p_{N}}\right)$ and operators $\left(\hat{q}^{1}, \ldots, \hat{q}^{N}\right)$, $\left(\hat{p}_{1}, \ldots, \hat{p}_{N}\right)$ will be simply denoted by $Q, P, \partial_{x}, \partial_{p}, \hat{q}, \hat{p}$. Also we define products of these sequences with vectors and matrices in a standard way.

The Moyal quantization scheme is as follows. First, let us assume that $M=\mathbb{R}^{2 N}$ and $\mathcal{P}=\partial_{x^{i}} \wedge \partial_{p_{i}}$. Define a $\star$-product by a formula

$$
f \star g=f \exp \left(\frac{1}{2} i \hbar \overleftarrow{\partial}_{x^{i}} \vec{\partial}_{p_{i}}-\frac{1}{2} i \hbar \overleftarrow{\partial}_{p_{i}} \vec{\partial}_{x^{i}}\right) g
$$

This $\star$-product is called the Moyal product. For a two-dimensional case $(N=1)$ the Moyal product reads

$$
f \star g=\sum_{k=0}^{\infty} \frac{1}{k !}\left(\frac{i \hbar}{2}\right)^{k} \sum_{m=0}^{k}\left(\begin{array}{c}
k \\
m
\end{array}\right)(-1)^{m}\left(\partial_{x}^{k-m} \partial_{p}^{m} f\right)\left(\partial_{x}^{m} \partial_{p}^{k-m} g\right) \text {. }
$$

The deformed Poisson bracket $\llbracket \cdot, \cdot \rrbracket$ associated with the $\star$-product will be given in terms of a $\star$-commutator $[\cdot, \cdot]$ as follows

$$
\llbracket f, g \rrbracket=\frac{1}{i \hbar}[f, g]=\frac{1}{i \hbar}(f \star g-g \star f), \quad f, g \in \mathcal{A}_{Q} .
$$

To avoid problems with convergence of the series in the above definition of the $\star$-product the common practise is to extend the space $C^{\infty}(M)$ to a space $C^{\infty}(M) \llbracket \hbar \rrbracket$ of formal power series in $\hbar$ with coefficients from $C^{\infty}(M)$. The $\star$-product is then properly defined on such space. Other approach is to define a $\star$-product on the space $\mathcal{S}\left(\mathbb{R}^{2 N}\right)$ of Schwartz functions using an integral formula and then extend it to an appropriate space of distributions [27-30]. It is then possible to define a topology on such space of distributions for which the $\star$-product, for a certain class of functions, can be expressed as the above series.

Note, that the Moyal quantization was performed in a specific canonical coordinate system, namely, in a Cartesian coordinate system on $\mathbb{R}^{2 N}$. In Section 3 we will see how to quantize systems in different coordinate systems.

Let us prove a property of functions from $\mathcal{A}_{Q}$ which will be used later on. For simplicity we only present the theorem for the case $N=1$. 
Theorem 2.1. Every function $f \in \mathcal{A}_{Q}$ can be expanded into a $a$-power series

$$
f=\sum_{n, m=0}^{\infty} a_{n m} \underbrace{x \star \cdots \star x}_{n} \star \underbrace{p \star \cdots \star p}_{m}
$$

where $a_{n m} \in \mathbb{C}$.

Proof. From recurrence relations

$$
\begin{aligned}
& x \cdot(\underbrace{x \star \cdots \star x}_{n} \star \underbrace{p \star \cdots \star p}_{m})=\underbrace{x \star \cdots \star x}_{n+1} \star \underbrace{p \star \cdots \star p}_{m}-\frac{1}{2} i \hbar m \underbrace{x \star \cdots \star x}_{n} \star \underbrace{p \star \cdots \star p}_{m-1}, \\
& p \cdot(\underbrace{x \star \cdots \star x}_{n} \star \underbrace{p \star \cdots \star p}_{m})=\underbrace{x \star \cdots \star x}_{n} \star \underbrace{p \star \cdots \star p}_{m+1}-\frac{1}{2} i \hbar n \underbrace{x \star \cdots \star x}_{n-1} \underbrace{p \star \cdots \star p}_{m}
\end{aligned}
$$

it follows that monomials $x^{n} p^{m}$ can be written as $\star$-polynomials. Thus after expanding $f$ into the Taylor series it is easily seen that $f$ can be written in the form (3).

\subsection{Space of states}

In general a space of states is fully characterized by the algebraic structure of the quantum Poisson algebra $\mathcal{A}_{Q}$ [31, 32]. It can be shown that for the Moyal quantization states can be represented as quantum distribution functions, i.e., square integrable functions $\Psi$ defined on the phase space satisfying certain conditions [26, 33]. For this reason the Hilbert space $\mathcal{H}=L^{2}\left(\mathbb{R}^{2 N}\right)$ of square integrable functions on the phase space will be called a space of states. Observe, that the Moyal product can be extended to a product between smooth functions from $C^{\infty}\left(\mathbb{R}^{2 N}\right)$ and square integrable functions from $L^{2}\left(\mathbb{R}^{2 N}\right)$. It is also possible to define the Moyal product between square integrable functions from $L^{2}\left(\mathbb{R}^{2 N}\right)$ by extending it from the space $\mathcal{S}\left(\mathbb{R}^{2 N}\right)$ of Schwartz functions [26].

\subsection{Observables and states as operators}

In Moyal quantization scheme observables $A \in \mathcal{A}_{Q}$ and states $\Psi \in \mathcal{H}$ can be treated as operators $\hat{A}$ and $\hat{\Psi}$ defined on the Hilbert space of states $\mathcal{H}$ by the following prescription

$$
\hat{A}=A \star, \quad \hat{\Psi}=(2 \pi \hbar)^{N / 2} \Psi \star .
$$

It can be proved that the operator $\hat{A}$ can be written as appropriately ordered function $A$ of operators of position $\left(\hat{q}_{M}\right)^{i}=x^{i} \star=x^{i}+\frac{1}{2} i \hbar \partial_{p_{j}}$ and momentum $\left(\hat{p}_{M}\right)_{j}=p_{j} \star=p_{j}-\frac{1}{2} i \hbar \partial_{x^{i}}\left(\left[\left(\hat{q}_{M}\right)^{i},\left(\hat{p}_{M}\right)_{j}\right]=i \hbar \delta_{j}^{i}\right)$

$$
\hat{A}=A \star=A_{M}\left(\hat{q}_{M}, \hat{p}_{M}\right) .
$$

$A_{M}(\hat{q}, \hat{p})$ denotes symmetrically-ordered function of operators $\hat{q}$ and $\hat{p}$.

Note that the Hermitian conjugation $\dagger$ of the operators $\hat{A}$ and $\hat{\Psi}$ in Moyal quantization scheme is related to the complex-conjugation $*$ (involution in $\mathcal{A}_{Q}$ ) by the formulae

$$
\hat{A}^{\dagger}=(A \star)^{\dagger}=A^{*} \star, \quad \hat{\Psi}^{\dagger}=\left((2 \pi \hbar)^{N / 2} \Psi \star\right)^{\dagger}=(2 \pi \hbar)^{N / 2} \Psi^{*} \star
$$

\subsection{Expectation values of observables and time evolution equation}

Formulas for the expectation values of observables and the time evolution of states are similar as in classical mechanics, except that the point-wise product $\cdot$ of functions and the Poisson bracket $\{\cdot, \cdot\}$ have to be replaced with the $\star$-product and the quantum Poisson bracket $\llbracket \cdot, \cdot \rrbracket$. Thus, the expectation value of an observable $A \in \mathcal{A}_{Q}$ in a state $\Psi \in \mathcal{H}$ is given by the formula

$$
\begin{aligned}
\langle A\rangle_{\Psi} & =\frac{1}{(2 \pi \hbar)^{N / 2}} \iint(A(0) \star \Psi(t))(x, p) \mathrm{d} x \mathrm{~d} p=\langle A(0)\rangle_{\Psi(t)} \\
& =\frac{1}{(2 \pi \hbar)^{N / 2}} \iint(A(t) \star \Psi(0))(x, p) \mathrm{d} x \mathrm{~d} p=\langle A(t)\rangle_{\Psi(0)} .
\end{aligned}
$$


The time evolution equation of quantum distribution functions $\Psi(t)$ (Schrödinger picture) is the counterpart of the Liouville's equation describing the time evolution of classical distribution functions, and is given by the formula

$$
\frac{\mathrm{d} \Psi}{\mathrm{d} t}-\llbracket H, \Psi(t) \rrbracket=0 \quad \Leftrightarrow \quad i \hbar \frac{\mathrm{d} \Psi}{\mathrm{d} t}-[H, \Psi(t)]=0,
$$

where $H$ is a Hamiltonian (distinguished real function from $\mathcal{A}_{Q}$ ). The time evolution of quantum observable $A(t)$ (Heisenberg picture) is given by

$$
\frac{\mathrm{d} A}{\mathrm{~d} t}-\llbracket A(t), H \rrbracket=0 \quad \Leftrightarrow \quad i \hbar \frac{\mathrm{d} A}{\mathrm{~d} t}-[A(t), H]=0 .
$$

\subsection{Equivalence of quantizations}

Two star-products $\star$ and $\star^{\prime}$ are said to be gauge equivalent if there exists a vector space automorphism $S: C^{\infty}\left(\mathbb{R}^{2 N}\right) \rightarrow C^{\infty}\left(\mathbb{R}^{2 N}\right)$ of the form

$$
S=\sum_{k=0}^{\infty} \hbar^{k} S_{k}, \quad S_{0}=1,
$$

where $S_{k}$ are linear operators, which satisfies the formula

$$
S(f \star g)=S f \star^{\prime} S g \quad f, g \in C^{\infty}\left(\mathbb{R}^{2 N}\right) .
$$

If, moreover, the automorphism $S$ preserves the deformed Poisson brackets and involutions $*$ and $*^{\prime}$ from the algebras $\mathcal{A}_{Q}=\left(C^{\infty}\left(\mathbb{R}^{2 N}\right), \star, \llbracket \cdot, \cdot \rrbracket, *\right)$ and $\mathcal{A}_{Q}^{\prime}=\left(C^{\infty}\left(\mathbb{R}^{2 N}\right), \star^{\prime}, \llbracket \cdot, \cdot \rrbracket^{\prime}, *^{\prime}\right)$, i.e.,

$$
\begin{aligned}
S(\llbracket f, g \rrbracket) & =\llbracket S f, S g \rrbracket^{\prime}, \\
S\left(f^{*}\right) & =(S f)^{*^{\prime}},
\end{aligned}
$$

then $S$ is an isomorphism of the algebra $\mathcal{A}_{Q}$ onto the algebra $\mathcal{A}_{Q}^{\prime}$.

Two quantizations of a classical Hamiltonian system are equivalent if there exists an isomorphism $S$ of their quantum Poisson algebras. This equivalence is mathematical as well as physical. It has been stressed out in Subsection 2.1, that to the same measurable quantity correspond different functions from respective quantum Poisson algebras. This observation seems to be missing in considerations of different quantizations present in the literature. In fact, to every observable $A \in \mathcal{A}_{Q}$ from one quantization scheme corresponds an observable $A^{\prime}=S A \in \mathcal{A}_{Q}^{\prime}$ from the other quantization scheme. Both observables $A$ and $A^{\prime}$ describe the same measurable quantity and in the limit $\hbar \rightarrow 0$ reduce to the same classical observable. If, for example, the initial quantization is the Moyal one, then quantum observables are real-valued functions and as a quantum observable $A$ we can take a related classical observable $A_{C}$, i.e., $A=A_{C}$ and is $\hbar$-independent. Then, in some other quantization scheme, gauge equivalent to the Moyal one, to the same observable $A$ corresponds another function $A^{\prime}=S A_{C}$, $\hbar$-dependent and complex in general. Such approach to equivalence of quantum systems introduces, indeed, physically equivalent quantizations as the functions $A, A^{\prime}$ from different quantization schemes have the same spectra, expectation values, etc., and when they are Hamiltonians they describe the same time evolution.

The presented formalism of phase space quantum mechanics can be extended to a very broad class of quantization schemes equivalent to the Moyal-quantization scheme. Let $S$ be a vector space automorphism of $C^{\infty}\left(\mathbb{R}^{2 N}\right)$ of the form (5). Moreover, lets assume that $S$ satisfies

$$
\begin{gathered}
S x^{i}=x^{i}, \quad S p_{j}=p_{j}, \\
S\left(f^{*}\right)=(S f)^{*} .
\end{gathered}
$$

The automorphism $S$ defines a new star-product equivalent to the Moyal-product by the equation

$$
f \star_{S} g:=S\left(S^{-1} f \star S^{-1} g\right), \quad f, g \in C^{\infty}\left(\mathbb{R}^{2 N}\right),
$$


with involution being also the complex-conjugation. We will show that such class of equivalent quantization schemes is related to the choice of different quantum canonical coordinates for quantum systems.

It is possible to define a morphism of spaces of states of different quantization schemes, in terms of $S$. This morphism we will also denote by $S$. In case when the initial quantization is the Moyal quantization $S$ will be a Hilbert space isomorphism. In what follows we will restrict to the case when the $S$-image of the space of states $L^{2}\left(\mathbb{R}^{2 N}\right)$ is also a Hilbert space $L^{2}\left(\mathbb{R}^{2 N}, \mu\right)$ of square integrable functions possibly with respect to a different measure $\mu$, and when $S$ vanishes under the integral sign, i.e.,

$$
\iint S \Psi(x, p) \mathrm{d} \mu(x, p)=\iint \Psi(x, p) \mathrm{d} x \mathrm{~d} p
$$

for every quantum distribution function $\Psi$.

All previous results for the Moyal-quantization scheme are also valid for the $S$-quantization scheme. In particular, the symmetrically-ordered operator function $A_{M}(\hat{q}, \hat{p})$ of operators $\hat{q}^{i}, \hat{p}_{j}$ has to be replaced by the following $S$-ordering

$$
A_{S}(\hat{q}, \hat{p}):=\left(S^{-1} A\right)_{M}(\hat{q}, \hat{p}) .
$$

Also the following result holds true [26]

$$
A \star_{S}=A_{S}\left(\hat{q}_{S}, \hat{p}_{S}\right),
$$

where

$$
\begin{aligned}
& \left(\hat{q}_{S}\right)^{i}:=x^{i} \star_{S}=S\left(\hat{q}_{M}\right)^{i} S^{-1}, \\
& \left(\hat{p}_{S}\right)_{j}:=p_{j} \star_{S}=S\left(\hat{p}_{M}\right)_{j} S^{-1} .
\end{aligned}
$$

The above results are important for transformation theory develop in the next section.

\subsection{Ordinary description of quantum mechanics}

The phase space quantum mechanics is equivalent to the ordinary description of quantum mechanics. To show this first note that the Hilbert space of states can be written as the following tensor product of the Hilbert space $L^{2}\left(\mathbb{R}^{N}\right)$ and a space dual to it $\left(L^{2}\left(\mathbb{R}^{N}\right)\right)^{*}$ :

$$
\mathcal{H}=\left(L^{2}\left(\mathbb{R}^{N}\right)\right)^{*} \otimes_{S} L^{2}\left(\mathbb{R}^{N}\right)
$$

where the tensor product $\otimes_{S}$ is defined by

$$
\begin{aligned}
\left(\varphi^{*} \otimes_{S} \psi\right)(x, p) & =\frac{1}{(2 \pi \hbar)^{N / 2}} S \int \mathrm{d} y e^{-\frac{i}{\hbar} p y} \varphi^{*}\left(x-\frac{1}{2} y\right) \psi\left(x+\frac{1}{2} y\right) \\
& =\frac{1}{(2 \pi \hbar)^{N / 2}} \iiint \mathrm{d} x^{\prime} \mathrm{d} p^{\prime} \mathrm{d} y \varphi^{*}\left(x^{\prime}-\frac{1}{2} y\right) \psi\left(x^{\prime}+\frac{1}{2} y\right) S\left(x, p, x^{\prime}, p^{\prime}\right) e^{-\frac{i}{\hbar} p^{\prime} y},
\end{aligned}
$$

where $\varphi, \psi \in L^{2}\left(\mathbb{R}^{N}\right)$ and $S\left(x, p, x^{\prime}, p^{\prime}\right)$ is an integral kernel of the isomorphism $S$.

States $\Psi \in \mathcal{H}$ treated as operators $\hat{\Psi}=(2 \pi \hbar)^{N / 2} \Psi \star_{S}$ can be written in the following form

$$
\hat{\Psi}=\hat{1} \otimes_{S} \hat{\rho},
$$

where $\hat{\rho}$ is some density operator. Hence to every pure or mixed state $\Psi \in \mathcal{H}$ corresponds a unique density operator $\hat{\rho}$. Similarly, observables $A \in \mathcal{A}_{Q}$ treated as operators $\hat{A}=A \star_{S}$ take the form

$$
\hat{A}=A \star_{S}=\hat{1} \otimes_{S} A_{S}(\hat{q}, \hat{p}),
$$

where $\hat{q}^{i}=x^{i}$ and $\hat{p}_{j}=-i \hbar \partial_{x^{j}}$ are standard operators of position and momentum. In particular, from this it follows that

$$
A \star_{S} \Psi=\varphi^{*} \otimes_{S} A_{S}(\hat{q}, \hat{p}) \psi
$$


for $\Psi=\varphi^{*} \otimes_{S} \psi$ and $\varphi, \psi \in L^{2}\left(\mathbb{R}^{N}\right)$.

The expectation values of observables $A \in \mathcal{A}_{Q}$ in states $\Psi \in \mathcal{H}$ are the same as when computed in ordinary quantum mechanics

$$
\langle A\rangle_{\Psi}=\operatorname{tr}\left(\hat{\rho} A_{S}(\hat{q}, \hat{p})\right),
$$

where $\hat{\rho}$ is a density operator corresponding to $\Psi$ and $A_{S}(\hat{q}, \hat{p})$ is an operator corresponding to $A$. Also the time evolution equation of states $\Psi \in \mathcal{H}$ corresponds to the von Neumann equation describing the time evolution of density operators $\hat{\rho}$.

\section{Canonical transformations of coordinates in phase space quantum mechanics}

\subsection{General theory}

As before we will consider the Moyal quantization of a classical Hamiltonian system $(M, \mathcal{P}, H)$, where $M=\mathbb{R}^{2 N}, \mathcal{P}=\partial_{x^{i}} \wedge \partial_{p_{i}}$, and $H \in C^{\infty}(M)$ is an arbitrary real function. A transformation of phase space coordinates of the quantum system is defined as in classical mechanics, i.e., as a smooth bijective map $T: \mathbb{R}^{2 N} \supset U \ni\left(x^{\prime}, p^{\prime}\right) \rightarrow(x, p) \in W \subset \mathbb{R}^{2 N}$. For simplicity only transformations defined on almost the whole phase space will be considered, i.e., it will be assumed that $\mathbb{R}^{2 N} \backslash U$ and $\mathbb{R}^{2 N} \backslash W$ are sets of the Lebesgue-measure zero. A transformation $T$ transforms every observable $A \in C^{\infty}\left(\mathbb{R}^{2 N}\right)$ and every state $\Psi \in L^{2}\left(\mathbb{R}^{2 N}\right)$ by the formulae

$$
A^{\prime}=A \circ T, \quad \Psi^{\prime}=\Psi \circ T .
$$

In addition, also the Moyal-product needs to be transformed. The transformed star-product, denoted hereafter by $\star_{T}^{\prime}$, should fulfill the following natural condition

$$
(f \star g) \circ T=(f \circ T) \star_{T}^{\prime}(g \circ T), \quad f, g \in \mathcal{A}_{Q} .
$$

The $\star_{T}^{\prime}$-product is, in fact, given by the following formula

$$
f \star_{T}^{\prime} g=f \exp \left(\frac{1}{2} i \hbar \overleftarrow{D_{x^{\prime}}} \overrightarrow{D_{p_{j}^{\prime}}}-\frac{1}{2} i \hbar \overleftarrow{D_{p_{j}^{\prime}}} \overrightarrow{D_{x^{\prime}}}\right) g
$$

where vector fields $D_{x^{\prime i}}, D_{p_{j}^{\prime}}$ are derivations $\partial_{x^{i}}, \partial_{p_{j}}$ transformed by the transformation $T$ according to the rule

$$
\begin{aligned}
& \left(\partial_{x^{i}} f\right) \circ T=D_{x^{\prime}}(f \circ T), \quad f \in C^{\infty}\left(\mathbb{R}^{2 N}\right), \\
& \left(\partial_{p_{j}} f\right) \circ T=D_{p_{j}^{\prime}}(f \circ T), \quad f \in C^{\infty}\left(\mathbb{R}^{2 N}\right) .
\end{aligned}
$$

Notice, that a new product defined by vector fields $D_{x^{\prime i}}, D_{p_{j}^{\prime}}$ is well defined star-product, which associativity follows from commutativity of $D_{x^{\prime i}}$ and $D_{p_{j}^{\prime}}$.

To this moment we considered general transformations of coordinates. In what follows we will focus on an important class of transformations, namely, canonical transformations. In classical mechanics a canonical transformation is such transformation $T$ of phase space coordinates which transforms the system from one canonical coordinate system to the other. In other words, $T$ is a canonical transformation if it preserves the form of the Poisson bracket, i.e.,

$$
\left\{x^{\prime i}, p_{j}^{\prime}\right\}^{\prime}=\delta_{j}^{i},
$$

where $\{\cdot, \cdot\}^{\prime}$ denotes a Poisson bracket transformed by $T$ to the new coordinate system:

$$
\{f, g\}^{\prime}=\left\{f \circ T^{-1}, g \circ T^{-1}\right\} \circ T, \quad f, g \in C^{\infty}\left(\mathbb{R}^{2 N}\right) .
$$

Canonical transformations in phase space quantum mechanics are defined in a similar manner. Namely, a quantum canonical transformation is such transformation $T$ of coordinates which preserves the form of the deformed Poisson bracket, i.e.,

$$
\llbracket x^{\prime i}, p_{j}^{\prime} \rrbracket^{\prime}=\delta_{j}^{i},
$$


where $\llbracket \cdot, \cdot \rrbracket^{\prime}$ denotes a deformed Poisson bracket transformed by $T$ to the new coordinate system:

$$
\llbracket f, g \rrbracket^{\prime}=\llbracket f \circ T^{-1}, g \circ T^{-1} \rrbracket \circ T, \quad f, g \in C^{\infty}\left(\mathbb{R}^{2 N}\right) .
$$

In what follows we will consider only transformations $T$ which are quantum canonical transformations, with a special attention to the subclass of these which are also classically canonical, because the theory takes the simplest form for such class of transformations.

Now we take the advantage from the following property of a transformed star-product, valid for a wide class of quantum canonical transformations as we will see in Section 5. For any quantum canonical coordinate transformation $T$ with respect to a given $\star$-product, there exists a unique linear automorphism $S_{T}$ of the algebra $\mathcal{A}_{Q}$ in the form (5) satisfying

$$
\begin{gathered}
S_{T}\left(f \star^{\prime} g\right)=\left(S_{T} f\right) \star_{T}^{\prime}\left(S_{T} g\right), \quad f, g \in \mathcal{A}_{Q}, \\
S_{T} x^{\prime i}=x^{\prime i}, \quad S_{T} p_{j}^{\prime}=p_{j}^{\prime}, \\
S_{T}\left(f^{*}\right)=\left(S_{T} f\right)^{*}, \quad f \in \mathcal{A}_{Q},
\end{gathered}
$$

where $\star^{\prime}$ is the initial product expressed in $x^{\prime}, p^{\prime}$ variables

$$
f \star^{\prime} g=f \exp \left(\frac{1}{2} i \hbar \overleftarrow{\partial}_{x^{\prime i}} \vec{\partial}_{p_{i}^{\prime}}-\frac{1}{2} i \hbar \overleftarrow{\partial}_{p_{i}^{\prime}} \vec{\partial}_{x^{\prime i}}\right) g
$$

We will consider only such transformations for which the above statement is true, however, we believe that this statement holds for every quantum canonical transformation. The above property will play a crucial role while passing to the ordinary description of quantum mechanics. From (6) it is clear that two quantum Hamiltonian systems related to each other by some quantum canonical change of phase space coordinates are gauge equivalent.

For deriving the form of the automorphism $S_{T}$ for particular transformations $T$, other form of the conditions (6) will be more useful.

Theorem 3.1. The conditions (6) are fulfilled if and only if the conditions

$$
\begin{aligned}
\left(\hat{q}_{T}^{\prime}\right)^{i} & =S_{T}\left(\hat{q}_{M}\right)^{i} S_{T}^{-1}, \\
\left(\hat{p}_{T}^{\prime}\right)_{j} & =S_{T}\left(\hat{p}_{M}\right)_{j} S_{T}^{-1},
\end{aligned}
$$

are fulfilled, where $\left(\hat{q}_{T}^{\prime}\right)^{i}=x^{\prime i} \star_{T}^{\prime},\left(\hat{p}_{T}^{\prime}\right)_{j}=p_{j}^{\prime} \star_{T}^{\prime},\left(\hat{q}_{M}\right)^{i}=x^{\prime i} \star^{\prime}$ and $\left(\hat{p}_{M}\right)_{j}=p_{j}^{\prime} \star^{\prime}$.

Proof. For simplicity we will present the proof for a two-dimensional case $(N=1)$. If the conditions (6) are fulfilled then trivially the conditions (7) are fulfilled. Assume now, that the conditions (7) are fulfilled. From (77) it follows that (6a) will be satisfied for every $f$ in the form of a $\star^{\prime}$-monomial $x^{\prime} \star^{\prime} \cdots \star^{\prime} x^{\prime} \star^{\prime} p^{\prime} \star^{\prime} \cdots \star^{\prime} p^{\prime}$ as

$$
\begin{aligned}
S_{T}( & \left.(\underbrace{x^{\prime} \star^{\prime} \cdots \star^{\prime} x^{\prime} \star^{\prime}}_{n} \underbrace{p^{\prime} \star^{\prime} \cdots \star^{\prime} p^{\prime}}_{m}) \star^{\prime} g\right)=S_{T} x^{\prime} \star_{T}^{\prime} S_{T}(\underbrace{x^{\prime} \star^{\prime} \cdots \star^{\prime} x^{\prime}}_{n-1} \star^{\prime} \underbrace{p^{\prime} \star^{\prime} \cdots \star^{\prime} p^{\prime} \star^{\prime} g}_{m}) \\
& =\underbrace{S_{T} x^{\prime} \star_{T}^{\prime} \cdots \star_{T}^{\prime} S_{T} x^{\prime}}_{n} \star_{T}^{\prime} \underbrace{S_{T} p^{\prime} \star_{T}^{\prime} \cdots \star_{T}^{\prime} S_{T} p^{\prime} \star_{T}^{\prime} S_{T} g}_{m} \\
& =\underbrace{S_{T} x^{\prime} \star_{T}^{\prime} \cdots \star_{T}^{\prime} S_{T} x^{\prime}}_{m} \star_{T}^{\prime} \underbrace{S_{T} p^{\prime} \star_{T}^{\prime} \cdots \star_{T}^{\prime} S_{T} p^{\prime} \star_{T}^{\prime} S_{T}\left(p^{\prime} \star^{\prime} p^{\prime}\right) \star_{T}^{\prime} S_{T} g}_{m-2} \\
& =S_{T}(\underbrace{x^{\prime} \star^{\prime} \cdots \star^{\prime} x^{\prime}}_{n} \star^{\prime} \underbrace{p^{\prime} \star^{\prime} \cdots \star^{\prime} p^{\prime}}_{m}) \star_{T}^{\prime} S_{T} g .
\end{aligned}
$$

From the linearity of $S_{T}$ and the fact that the general $f \in \mathcal{A}_{Q}$ can be written in the form of the series (3) follows the condition (6a). The condition (6b) can be received by calculating left and right sides of (7) on function identically equal 1 . Furthermore, the condition (6c) can be proved in a similar manner as (6a) noting that the complex-conjugation is an involution for the $\star^{\prime}$ and $\star_{T}^{\prime}$-products, and using (6b). 
Note that every transformation $T$ of coordinates preserves the involution of the algebra $\mathcal{A}_{Q}$ (complex conjugation). Indeed, there holds

$$
(f \circ T)^{*}=f^{*} \circ T, \quad f \in \mathcal{A}_{Q} .
$$

We prove the property (6c) of the isomorphism $S_{T}$. Namely,

Theorem 3.2. For every quantum canonical transformation $T$ the isomorphism $S_{T}:\left(C^{\infty}\left(\mathbb{R}^{2 N}\right), \star^{\prime}, *\right) \rightarrow$ $\left(C^{\infty}\left(\mathbb{R}^{2 N}\right), \star_{T}^{\prime}, *\right)$ preserves the involution, i.e.,

$$
S_{T}\left(f^{*}\right)=\left(S_{T} f\right)^{*}, \quad f \in C^{\infty}\left(\mathbb{R}^{2 N}\right) .
$$

In other words $S_{T}=S_{T}^{*}$.

Proof. Using the fact that $S_{T}$ is an isomorphism of the space of states $\mathcal{H}$ onto the transformed space of states $\mathcal{H}^{\prime}$ (see below) the following equality holds

$$
\begin{aligned}
\left\langle\Phi \mid f^{*} \star^{\prime} \Psi\right\rangle_{\mathcal{H}} & =\left\langle\Phi \mid\left(f \star^{\prime}\right)^{\dagger} \Psi\right\rangle_{\mathcal{H}}=\left\langle f \star^{\prime} \Phi \mid \Psi\right\rangle_{\mathcal{H}}=\left\langle S_{T}\left(f \star^{\prime} \Phi\right) \mid S_{T} \Psi\right\rangle_{\mathcal{H}^{\prime}}=\left\langle S_{T} f \star_{T}^{\prime} S_{T} \Phi \mid S_{T} \Psi\right\rangle_{\mathcal{H}^{\prime}} \\
& =\left\langle S_{T} \Phi \mid\left(S_{T} f \star_{T}^{\prime}\right)^{\dagger} S_{T} \Psi\right\rangle_{\mathcal{H}^{\prime}}=\left\langle S_{T} \Phi \mid\left(S_{T} f\right)^{*} \star_{T}^{\prime} S_{T} \Psi\right\rangle_{\mathcal{H}^{\prime}}=\left\langle\Phi \mid S_{T}^{-1}\left(S_{T} f\right)^{*} \star^{\prime} \Psi\right\rangle_{\mathcal{H}}
\end{aligned}
$$

for every $\Phi, \Psi \in \mathcal{H}$. From this follows that $f^{*}=S_{T}^{-1}\left(S_{T} f\right)^{*}$.

It means that for every quantum canonical transformation $T$ the isomorphism $S_{T}$ generates new quantization scheme, equivalent to the Moyal one, with involution being also complex conjugation.

A transformation $T$ of coordinates transforms the Hilbert space of states $L^{2}\left(\mathbb{R}^{2 N}\right)$ into the Hilbert space $L^{2}\left(\mathbb{R}^{2 N}, \mu_{T}\right)$ with the standard scalar product, where $\mathrm{d} \mu_{T}\left(x^{\prime}, p^{\prime}\right)=\left|\operatorname{det} T^{\prime}\left(x^{\prime}, p^{\prime}\right)\right| \mathrm{d} x^{\prime} \mathrm{d} p^{\prime}$. Of course, if $T$ is also a classical canonical transformation then $\mathrm{d} \mu_{T}\left(x^{\prime}, p^{\prime}\right)=\mathrm{d} x^{\prime} \mathrm{d} p^{\prime}$, since the Jacobian of a classical canonical transformation is equal 1. The following property of $S_{T}$ has to hold to ensure the consistency of the whole approach to transformations of coordinates:

For a transformation $T$ of coordinates

$$
S_{T}: L^{2}\left(\mathbb{R}^{2 N}\right) \rightarrow L^{2}\left(\mathbb{R}^{2 N}, \mu_{T}\right)
$$

and $S_{T}$ is a Hilbert space isomorphism. Hence the space $L^{2}\left(\mathbb{R}^{2 N}, \mu_{T}\right)$ is a properly defined space of states after the transformation of coordinates. Moreover, the isomorphism $S_{T}$ vanishes under the integral sign, i.e.,

$$
\iint f\left(x^{\prime}, p^{\prime}\right) \mathrm{d} x^{\prime} \mathrm{d} p^{\prime}=\iint S_{T} f\left(x^{\prime}, p^{\prime}\right) \mathrm{d} \mu\left(x^{\prime}, p^{\prime}\right)
$$

Thus we will consider only such transformations for which the above property is fulfilled, however, we believe that every quantum canonical transformation satisfies the above property. Observe that a classically canonical transformation $T$ transforms the space of states $L^{2}\left(\mathbb{R}^{2 N}\right)$ onto itself. Thus $S_{T}$ in this case is an isomorphism (unitary operator) on $L^{2}\left(\mathbb{R}^{2 N}\right)$.

Canonical transformations appear as trajectories in phase space when one considers the time evolution of a system. This is true both in classical and quantum mechanics. In phase space quantum mechanics the solution of quantum Hamiltonian equations

$$
\dot{Q}^{i}(t)=\llbracket Q^{i}(t), H \rrbracket, \quad \dot{P}_{j}(t)=\llbracket P_{j}(t), H \rrbracket,
$$

where $Q^{i}(x, p, 0)=x^{i}$ and $P_{j}(x, p, 0)=p_{j}$, i.e., the Heisenberg representation (4) for observables of position and momentum, generates a quantum flow $\Phi_{t}$ in phase space according to an equation

$$
\Phi_{t}(x, p ; \hbar)=(Q(x, p, t ; \hbar), P(x, p, t ; \hbar)) .
$$

For every instance of time $t$ the map $\Phi_{t}$ is a quantum canonical transformation (quantum symplectomorphism) from coordinates $x, p$ to new coordinates $x^{\prime}=Q(x, p, t ; \hbar), p^{\prime}=P(x, p, t ; \hbar)$. Of course, in the limit $\hbar \rightarrow 0$ any quantum trajectory reduces to a classical trajectory. More thorough description of quantum trajectories we present in [34]. 


\subsection{Transformations which are both classical and quantum canonical}

The simplest subclass of quantum canonical transformations is generated by these quantum flows which coincide with classical flows. It means that the quantum canonical transformation (9) is $\hbar$ independent and coincides with classical symplectomorphism, i.e., is a simultaneous solution of quantum and classical Hamiltonian equations. Such transformations are generated from an appropriate generating function for any fixed value of the evolution parameter $t$.

In what follows we will present four important classes of nonlinear canonical transformations (both classical and quantum). The linear case is considered separately in Section 5 A sufficient condition for such class of transformations is a linearity in one set of arguments of an appropriate generating function. Let us begin with the case of two-dimensional phase space. The first class of transformations is determined by generating function of the form

$$
F_{1}\left(x, x^{\prime}\right)=x \phi_{1}\left(x^{\prime}\right)+\phi_{2}\left(x^{\prime}\right)
$$

with related transformations expressed by the equations

$$
p=\frac{\partial F_{1}}{\partial x}\left(x, x^{\prime}\right), \quad p^{\prime}=-\frac{\partial F_{1}}{\partial x^{\prime}}\left(x, x^{\prime}\right),
$$

where $\phi_{1}$ is some smooth bijective function and $\phi_{2}$ is some smooth function. The above equations give the transformation in a form

$$
T_{1}\left(x^{\prime}, p^{\prime}\right)=\left(-\left(\phi_{1}^{\prime}\left(x^{\prime}\right)\right)^{-1} p^{\prime}-\left(\phi_{1}^{\prime}\left(x^{\prime}\right)\right)^{-1} \phi_{2}^{\prime}\left(x^{\prime}\right), \phi_{1}\left(x^{\prime}\right)\right),
$$

where $\phi^{\prime}\left(x^{\prime}\right)=\frac{d}{d x^{\prime}} \phi\left(x^{\prime}\right)$. Note, that a generating function

$$
\tilde{F}_{1}\left(x, x^{\prime}\right)=-x^{\prime} \phi_{1}(x)-\phi_{2}(x)
$$

generates a transformation $\tilde{T}_{1}$ being an inverse transformation to $T_{1}$.

The second class is generated by a function

$$
F_{2}\left(p, p^{\prime}\right)=-p \phi_{1}\left(p^{\prime}\right)-\phi_{2}\left(p^{\prime}\right)
$$

with a related transformation expressed by the equations

$$
x=-\frac{\partial F_{2}}{\partial p}\left(p, p^{\prime}\right), \quad x^{\prime}=\frac{\partial F_{2}}{\partial p^{\prime}}\left(p, p^{\prime}\right),
$$

which give

$$
T_{2}\left(x^{\prime}, p^{\prime}\right)=\left(\phi_{1}\left(p^{\prime}\right),-\left(\phi_{1}^{\prime}\left(p^{\prime}\right)\right)^{-1} x^{\prime}-\left(\phi_{1}^{\prime}\left(p^{\prime}\right)\right)^{-1} \phi_{2}^{\prime}\left(p^{\prime}\right)\right) .
$$

In this case the generating function

$$
\tilde{F}_{2}\left(p, p^{\prime}\right)=p^{\prime} \phi_{1}(p)+\phi_{2}(p)
$$

generates a transformation $\tilde{T}_{2}$ being an inverse transformation to $T_{2}$.

The third and fourth classes of transformations are generated by functions

$$
\begin{aligned}
& F_{3}\left(x, p^{\prime}\right)=x \phi_{1}\left(p^{\prime}\right)+\phi_{2}\left(p^{\prime}\right), \\
& F_{4}\left(x^{\prime}, p\right)=-p \phi_{1}\left(x^{\prime}\right)-\phi_{2}\left(x^{\prime}\right),
\end{aligned}
$$

with related transformations expressed by the equations

$$
p=\frac{\partial F_{3}}{\partial x}\left(x, p^{\prime}\right), \quad x^{\prime}=\frac{\partial F_{3}}{\partial p^{\prime}}\left(x, p^{\prime}\right),
$$

and

$$
x=-\frac{\partial F_{4}}{\partial p}\left(x^{\prime}, p\right), \quad p^{\prime}=-\frac{\partial F_{4}}{\partial x^{\prime}}\left(x^{\prime}, p\right) .
$$


The above equations give the transformations in a form

$$
\begin{aligned}
& T_{3}\left(x^{\prime}, p^{\prime}\right)=\left(\left(\phi_{1}^{\prime}\left(p^{\prime}\right)\right)^{-1} x^{\prime}-\left(\phi_{1}^{\prime}\left(p^{\prime}\right)\right)^{-1} \phi_{2}^{\prime}\left(p^{\prime}\right), \phi_{1}\left(p^{\prime}\right)\right), \\
& T_{4}\left(x^{\prime}, p^{\prime}\right)=\left(\phi_{1}\left(x^{\prime}\right),\left(\phi_{1}^{\prime}\left(x^{\prime}\right)\right)^{-1} p^{\prime}-\left(\phi_{1}^{\prime}\left(x^{\prime}\right)\right)^{-1} \phi_{2}^{\prime}\left(x^{\prime}\right)\right) .
\end{aligned}
$$

In this case the generating functions

$$
\begin{aligned}
& \tilde{F}_{3}\left(x, p^{\prime}\right)=-p^{\prime} \phi_{1}(x)-\phi_{2}(x), \\
& \tilde{F}_{4}\left(x^{\prime}, p\right)=x^{\prime} \phi_{1}(p)+\phi_{2}(p)
\end{aligned}
$$

generate transformations $\tilde{T}_{3}=T_{4}^{-1}$ and $\tilde{T}_{4}=T_{3}^{-1}$.

Note, that the transformations $T_{2}, T_{3}$ and $T_{4}$ can be constructed from $T_{1}$ with the help of an interchange of variables transformation $I\left(x^{\prime}, p^{\prime}\right)=\left(-p^{\prime}, x^{\prime}\right)$ being a special case of the transformation $T_{1}$ generated by a function $F\left(x, x^{\prime}\right)=x x^{\prime}$ :

$$
\begin{aligned}
& T_{2}=I \circ T_{1} \circ I^{-1}, \\
& T_{3}=T_{1} \circ I^{-1}, \\
& T_{4}=I^{-1} \circ T_{1} .
\end{aligned}
$$

Thus instead of considering the transformation theory for transformations $T_{1}, T_{2}, T_{3}$ and $T_{4}$ it is enough to consider only the transformation $T_{1}$ and it appropriate compositions with $I$.

The four presented classes of transformations are obviously classically canonical. It can be shown that they are also quantum canonical.

The presented considerations can be easily extended to a $2 N$-dimensional case. As an example let us present the transformation (10) in $2 N$-dimensions. Consider a generating function

$$
F\left(x, x^{\prime}\right)=\sum_{i} x^{i} \phi_{1}^{i}\left(x^{\prime}\right)+\phi_{2}\left(x^{\prime}\right)
$$

where $\phi_{1}=\left(\phi^{1}, \ldots, \phi^{N}\right): \mathbb{R}^{N} \rightarrow \mathbb{R}^{N}$ is a smooth bijective function and $\phi_{2}: \mathbb{R}^{N} \rightarrow \mathbb{R}$ a smooth function, and $x^{\prime}=\left(x^{\prime 1}, \ldots, x^{\prime N}\right)$. The function $F$ generates the following transformation

$$
\begin{aligned}
T\left(x^{\prime}, p^{\prime}\right)= & \left(-\left(\phi_{1}^{\prime}\left(x^{\prime}\right)\right)^{-1} \cdot p^{\prime}-\left(\phi_{1}^{\prime}\left(x^{\prime}\right)\right)^{-1} \cdot \phi_{2}^{\prime}\left(x^{\prime}\right), \phi_{1}\left(x^{\prime}\right)\right) \\
= & \left(-\left(J_{\phi_{1}}^{-1}\left(x^{\prime}\right)\right)_{1}^{i} p_{i}^{\prime}-\left(J_{\phi_{1}}^{-1}\left(x^{\prime}\right)\right)_{1}^{i}\left(J_{\phi_{2}}\left(x^{\prime}\right)\right)_{i}, \cdots,-\left(J_{\phi_{1}}^{-1}\left(x^{\prime}\right)\right)_{N}^{i} p_{i}^{\prime}-\left(J_{\phi_{1}}^{-1}\left(x^{\prime}\right)\right)_{N}^{i}\left(J_{\phi_{2}}\left(x^{\prime}\right)\right)_{i},\right. \\
& \left.\phi_{1}^{1}\left(x^{\prime}\right), \ldots, \phi_{1}^{N}\left(x^{\prime}\right)\right),
\end{aligned}
$$

where $\left(J_{\phi_{1}}\right)_{i}^{j}=\frac{\partial \phi_{1}^{j}}{\partial x^{\prime i}}$ is a Jacobian matrix of the function $\phi_{1}=\left(\phi_{1}^{1}, \ldots, \phi_{1}^{N}\right)$ and $\left(J_{\phi_{2}}\right)_{i}=\frac{\partial \phi_{2}}{\partial x^{\prime i}}$ is a Jacobian matrix of $\phi_{2}$. A calculation shows that this transformation is also quantum canonical. All other cases can be extended in a similar fashion.

\subsection{Transformation of observables treated as operators}

Let us introduce the following operator $\hat{T}: \mathcal{H} \rightarrow \mathcal{H}^{\prime}$ of the Hilbert spaces of states $\mathcal{H}=L^{2}\left(\mathbb{R}^{2 N}\right)$ and $\mathcal{H}^{\prime}=L^{2}\left(\mathbb{R}^{2 N}, \mu_{T}\right)$ by the equation

$$
\hat{T} \Psi=\Psi \circ T, \quad \Psi \in \mathcal{H} .
$$

The operator $\hat{T}$ is obviously linear and bijective with the inverse given by

$$
\hat{T}^{-1} \Psi=\Psi \circ T^{-1}, \quad \Psi \in \mathcal{H}^{\prime} .
$$

Moreover, the operator $\hat{T}$ preserves the scalar product, hence it is an isomorphism of the Hilbert spaces of states. There holds the following theorem. 
Theorem 3.3. Let $T$ be some quantum canonical transformation of coordinates and $A \in \mathcal{A}_{Q}$. There holds

$$
\hat{T} A_{M}\left(\hat{q}_{M}, \hat{p}_{M}\right) \hat{T}^{-1}=A_{S_{T}}^{\prime}\left(\hat{q}_{T}^{\prime}, \hat{p}_{T}^{\prime}\right)
$$

where $A^{\prime}=A \circ T$ and

$$
\left(\hat{q}_{T}^{\prime}\right)^{i}=x^{i} \star_{T}^{\prime}, \quad\left(\hat{p}_{T}^{\prime}\right)_{j}=p_{j}^{\prime} \star_{T}^{\prime} .
$$

Proof. Let $\Psi \in \mathcal{H}$. Then there holds

$$
\hat{T} A_{M}\left(\hat{q}_{M}, \hat{p}_{M}\right) \Psi=\hat{T}(A \star \Psi)=A^{\prime} \star_{T}^{\prime} \hat{T} \Psi=A_{S_{T}}^{\prime}\left(\hat{q}_{T}^{\prime}, \hat{p}_{T}^{\prime}\right) \hat{T} \Psi .
$$

\section{Corollary 3.1.}

$$
\begin{aligned}
\left(\hat{q}_{T}^{\prime}\right)^{i} & =\hat{T}\left(Q^{i}\right)_{M}\left(\hat{q}_{M}, \hat{p}_{M}\right) \hat{T}^{-1}, \\
\left(\hat{p}_{T}^{\prime}\right)_{j} & =\hat{T}\left(P_{j}\right)_{M}\left(\hat{q}_{M}, \hat{p}_{M}\right) \hat{T}^{-1},
\end{aligned}
$$

or from the other side

$$
\begin{aligned}
\left(\hat{q}_{M}\right)^{i} & =\hat{T}^{-1}\left(q^{i}\right)_{S_{T}}\left(\hat{q}_{T}^{\prime}, \hat{p}_{T}^{\prime}\right) \hat{T}, \\
\left(\hat{p}_{M}\right)_{j} & =\hat{T}^{-1}\left(p_{j}\right)_{S_{T}}\left(\hat{q}_{T}^{\prime}, \hat{p}_{T}^{\prime}\right) \hat{T},
\end{aligned}
$$

where $T^{-1}(x, p)=(Q(x, p), P(x, p))$ and $T\left(x^{\prime}, p^{\prime}\right)=\left(q\left(x^{\prime}, p^{\prime}\right), p\left(x^{\prime}, p^{\prime}\right)\right)$.

\section{Transformations of coordinates in ordinary quantum mechanics}

\subsection{General theory}

In the following section we use the formalism of transformations of coordinates in phase space quantum mechanics, presented in previous section, to develop a theory of transformations in ordinary quantum mechanics. First, observe that the transformation $T$ of coordinates induces a unitary operator $\hat{U}_{T}: L^{2}\left(\mathbb{R}^{N}\right) \rightarrow$ $L^{2}\left(\mathbb{R}^{N}\right)$ defined on the Hilbert space of states of the ordinary quantum mechanics, which transforms vector states and observables to the new coordinate system. Such operator is defined by the following equation

$$
\hat{T}\left(\varphi^{*} \otimes_{M} \psi\right)=\left(\varphi^{*} \otimes_{M} \psi\right) \circ T=:\left(\hat{U}_{T} \varphi\right)^{*} \otimes_{S_{T}} \hat{U}_{T} \psi, \quad \varphi, \psi \in L^{2}\left(\mathbb{R}^{N}\right) .
$$

The operator $\hat{U}_{T}$ should be unitary to assure that after a transformation of coordinates states remain properly defined. We state this as an another assumption about the considered class of transformations.

From the definition of the operator $\hat{U}_{T}$ follows an important formula for the transformation of observables to the new coordinate system.

Theorem 4.1. For $A \in \mathcal{A}_{Q}$ and an arbitrary quantum canonical transformation $T$ of coordinates there holds

$$
A_{S_{T}}^{\prime}\left(\hat{q}^{\prime}, \hat{p}^{\prime}\right)=\hat{U}_{T} A_{M}(\hat{q}, \hat{p}) \hat{U}_{T}^{-1},
$$

where $A^{\prime}=A \circ T$ and

$$
\begin{aligned}
\left(\hat{q}^{i} \psi\right)(x) & =x^{i} \psi(x), & \left(\hat{p}_{j} \psi\right)(x) & =-i \hbar \frac{\partial \psi}{\partial x^{j}}(x), \\
\left(\hat{q}^{i} \psi^{\prime}\right)\left(x^{\prime}\right) & =x^{\prime i} \psi^{\prime}\left(x^{\prime}\right), & \left(\hat{p}_{j}^{\prime} \psi^{\prime}\right)\left(x^{\prime}\right) & =-i \hbar \frac{\partial \psi^{\prime}}{\partial x^{\prime j}}\left(x^{\prime}\right),
\end{aligned}
$$

for $\psi, \psi^{\prime} \in L^{2}\left(\mathbb{R}^{N}\right)$. 
Proof. Let $\Psi=\varphi^{*} \otimes_{M} \psi$. Then from one side

$$
(A \star \Psi) \circ T=A^{\prime} \star_{T}^{\prime} \hat{T} \Psi=\left(\hat{U}_{T} \varphi\right)^{*} \otimes_{S_{T}} A_{S_{T}}^{\prime}\left(\hat{q}^{\prime}, \hat{p}^{\prime}\right) \hat{U}_{T} \psi
$$

and from the other side

$$
\begin{aligned}
(A \star \Psi) \circ T & =\left(\varphi^{*} \otimes_{M} A_{M}(\hat{q}, \hat{p}) \psi\right) \circ T=\left(\hat{U}_{T} \varphi\right)^{*} \otimes_{S_{T}} \hat{U}_{T} A_{M}(\hat{q}, \hat{p}) \psi \\
& =\left(\hat{U}_{T} \varphi\right)^{*} \otimes_{S_{T}} \hat{U}_{T} A_{M}(\hat{q}, \hat{p}) \hat{U}_{T}^{-1} \hat{U}_{T} \psi
\end{aligned}
$$

Comparison of the above two formulae implies the result.

The above result shows that applying the Born's quantization rule to a transformed classical observable gives an operator unitarily equivalent with an operator corresponding to an untransformed classical observable, provided that the ordering of $\hat{q}^{\prime}, \hat{p}^{\prime}$ will be appropriately changed. This ensures the consistency of a standard quantization procedure since we can quantize a classical Hamiltonian system in whatever canonical coordinate system, provided that we use an appropriate ordering of operators of position and momentum.

\section{Corollary 4.1.}

$$
\begin{aligned}
\hat{q}^{i} & =\hat{U}_{T}\left(Q^{i}\right)_{M}(\hat{q}, \hat{p}) \hat{U}_{T}^{-1} \\
\hat{p}_{j}^{\prime} & =\hat{U}_{T}\left(P_{j}\right)_{M}(\hat{q}, \hat{p}) \hat{U}_{T}^{-1}
\end{aligned}
$$

or from the other side

$$
\begin{aligned}
& \hat{q}^{i}=\hat{U}_{T}^{-1}\left(q^{i}\right)_{S_{T}}\left(\hat{q}^{\prime}, \hat{p}^{\prime}\right) \hat{U}_{T}, \\
& \hat{p}_{j}=\hat{U}_{T}^{-1}\left(p_{j}\right)_{S_{T}}\left(\hat{q}^{\prime}, \hat{p}^{\prime}\right) \hat{U}_{T},
\end{aligned}
$$

where $T^{-1}(x, p)=(Q(x, p), P(x, p))$ and $T\left(x^{\prime}, p^{\prime}\right)=\left(q\left(x^{\prime}, p^{\prime}\right), p\left(x^{\prime}, p^{\prime}\right)\right)$.

The above result can be understood as follows. An operator of position corresponding to a new coordinate system can be simply defined as $Q_{M}(\hat{q}, \hat{p})$. This operator can be written in a position representation, i.e., as an operator of multiplication by coordinate variable. More precisely, there exist a unitary operator $\hat{U}$ such that

From previous result $\hat{U}=\hat{U}_{T}$.

$$
\hat{U} Q_{M}(\hat{q}, \hat{p}) \hat{U}^{-1}=x^{\prime}
$$

\subsection{The form of the operator $\hat{U}_{T}$}

Equations (15) can be used to derive the form of the operator $\hat{U}_{T}$ for considered class of transformations. Indeed, for a given transformation $T$ it is enough to find such unitary operator $\hat{U}_{T}$ which will satisfy (15). Such operator will be unique.

In what follows let us find the form of the unitary operator $\hat{U}_{T}$, in a two-dimensional case, for a transformation $T_{1}\left(x^{\prime}, p^{\prime}\right)=\left(-\left(\phi_{1}^{\prime}\left(x^{\prime}\right)\right)^{-1} p^{\prime}-\left(\phi_{1}^{\prime}\left(x^{\prime}\right)\right)^{-1} \phi_{2}^{\prime}\left(x^{\prime}\right), \phi_{1}\left(x^{\prime}\right)\right)$ generated by a function $F_{1}\left(x, x^{\prime}\right)=$ $x \phi_{1}\left(x^{\prime}\right)+\phi_{2}\left(x^{\prime}\right)$ from Subsection 3.2. One calculates that for $\hat{U}_{T_{1}}$ in the form

$$
\left(\hat{U}_{T_{1}} \varphi\right)\left(x^{\prime}\right)=\frac{1}{\sqrt{2 \pi \hbar}} \int \varphi(x)\left|\phi_{1}^{\prime}\left(x^{\prime}\right)\right|^{1 / 2} e^{-\frac{i}{\hbar}\left(x \phi_{1}\left(x^{\prime}\right)+\phi_{2}\left(x^{\prime}\right)\right)} \mathrm{d} x
$$

the inverse operator to $\hat{U}_{T_{1}}$ reads

$$
\left(\hat{U}_{T_{1}}^{-1} \varphi\right)(x)=\frac{1}{\sqrt{2 \pi \hbar}} \int \varphi\left(x^{\prime}\right)\left|\phi_{1}^{\prime}\left(x^{\prime}\right)\right|^{1 / 2} e^{\frac{i}{\hbar}\left(x \phi_{1}\left(x^{\prime}\right)+\phi_{2}\left(x^{\prime}\right)\right)} \mathrm{d} x^{\prime}
$$


and that (15) are indeed satisfied. To prove this note that $Q_{M}(\hat{q}, \hat{p})=\phi_{1}^{-1}(\hat{p})$. Then

$$
\begin{aligned}
\left(\hat{U}_{T_{1}} Q_{M}(\hat{q}, \hat{p}) \hat{U}_{T_{1}}^{-1} \varphi\right)\left(x^{\prime}\right)= & \frac{1}{2 \pi \hbar} \iint \phi_{1}^{-1}\left(-i \hbar \partial_{x}\right)\left(\varphi\left(x^{\prime \prime}\right)\left|\phi_{1}^{\prime}\left(x^{\prime \prime}\right)\right|^{1 / 2} e^{\frac{i}{\hbar}\left(x \phi_{1}\left(x^{\prime \prime}\right)+\phi_{2}\left(x^{\prime \prime}\right)\right)}\right) \\
& \times\left|\phi_{1}^{\prime}\left(x^{\prime}\right)\right|^{1 / 2} e^{-\frac{i}{\hbar}\left(x \phi_{1}\left(x^{\prime}\right)+\phi_{2}\left(x^{\prime}\right)\right)} \mathrm{d} x \mathrm{~d} x^{\prime \prime} \\
= & \frac{1}{2 \pi \hbar} \iint x^{\prime \prime} \varphi\left(x^{\prime \prime}\right)\left|\phi_{1}^{\prime}\left(x^{\prime \prime}\right) \phi_{1}^{\prime}\left(x^{\prime}\right)\right|^{1 / 2} e^{\frac{i}{\hbar}\left(\phi_{1}\left(x^{\prime \prime}\right)-\phi_{1}\left(x^{\prime}\right)\right) x} e^{\frac{i}{\hbar}\left(\phi_{2}\left(x^{\prime \prime}\right)-\phi_{2}\left(x^{\prime}\right)\right)} \mathrm{d} x \mathrm{~d} x^{\prime \prime} \\
= & \int x^{\prime \prime} \varphi\left(x^{\prime \prime}\right)\left|\phi_{1}^{\prime}\left(x^{\prime \prime}\right) \phi_{1}^{\prime}\left(x^{\prime}\right)\right|^{1 / 2} \delta\left(\phi_{1}\left(x^{\prime \prime}\right)-\phi_{1}\left(x^{\prime}\right)\right) e^{\frac{i}{\hbar}\left(\phi_{2}\left(x^{\prime \prime}\right)-\phi_{2}\left(x^{\prime}\right)\right)} \mathrm{d} x^{\prime \prime} .
\end{aligned}
$$

After introducing new variable $\tilde{x}^{\prime \prime}=\phi_{1}\left(x^{\prime \prime}\right)$ one gets that $\mathrm{d} x^{\prime \prime}=\left|\phi_{1}^{\prime}\left(\phi_{1}^{-1}\left(\tilde{x}^{\prime \prime}\right)\right)\right|^{-1} \mathrm{~d} \tilde{x}^{\prime \prime}$ and the above formula can be written in a form

$$
\begin{aligned}
\left(\hat{U}_{T_{1}} Q_{M}(\hat{q}, \hat{p}) \hat{U}_{T_{1}}^{-1} \varphi\right)\left(x^{\prime}\right)= & \int \phi_{1}^{-1}\left(\tilde{x}^{\prime \prime}\right) \varphi\left(\phi_{1}^{-1}\left(\tilde{x}^{\prime \prime}\right)\right)\left|\phi_{1}^{\prime}\left(\phi_{1}^{-1}\left(\tilde{x}^{\prime \prime}\right)\right)\right|^{1 / 2}\left|\phi_{1}^{\prime}\left(x^{\prime}\right)\right|^{1 / 2} \delta\left(\tilde{x}^{\prime \prime}-\phi_{1}\left(x^{\prime}\right)\right) \\
& \times e^{\frac{i}{\hbar}\left(\phi_{2}\left(\phi_{1}^{-1}\left(\tilde{x}^{\prime \prime}\right)\right)-\phi_{2}\left(x^{\prime}\right)\right)} \mathrm{d} \tilde{x}^{\prime \prime} \\
= & x^{\prime} \varphi\left(x^{\prime}\right) .
\end{aligned}
$$

Equation (15b) can be proved analogically. Note, that (16) and (17) can be written in a general form

$$
\begin{aligned}
& \left(\hat{U}_{T_{1}} \varphi\right)\left(x^{\prime}\right)=\frac{1}{\sqrt{2 \pi \hbar}} \int \varphi(x) \sqrt{\left|\frac{\partial^{2} F_{1}}{\partial x \partial x^{\prime}}\left(x, x^{\prime}\right)\right|} e^{-\frac{i}{\hbar} F_{1}\left(x, x^{\prime}\right)} \mathrm{d} x, \\
& \left(\hat{U}_{T_{1}}^{-1} \varphi\right)(x)=\frac{1}{\sqrt{2 \pi \hbar}} \int \varphi\left(x^{\prime}\right) \sqrt{\left|\frac{\partial^{2} F_{1}}{\partial x \partial x^{\prime}}\left(x, x^{\prime}\right)\right|} e^{\frac{i}{\hbar} F_{1}\left(x, x^{\prime}\right)} \mathrm{d} x^{\prime} .
\end{aligned}
$$

A direct calculations show that $\hat{U}_{T_{1}}$ is indeed a unitary operator on the Hilbert space $L^{2}(\mathbb{R})$.

Let us also find the form of the unitary operator $\hat{U}_{T}$, also in a two-dimensional case, for the point transformation $T_{4}\left(x^{\prime}, p^{\prime}\right)=\left(\phi_{1}\left(x^{\prime}\right),\left(\phi_{1}^{\prime}\left(x^{\prime}\right)^{-1} p^{\prime}-\left(\phi_{1}^{\prime}\left(x^{\prime}\right)^{-1} \phi_{2}^{\prime}\left(x^{\prime}\right)\right)\right.\right.$ generated by the function $F_{4}\left(x^{\prime}, p\right)=$ $-p \phi_{1}\left(x^{\prime}\right)-\phi_{2}\left(x^{\prime}\right)$. One calculates that $\hat{U}_{T_{4}}$ in the form

$$
\begin{aligned}
\left(\hat{U}_{T_{4}} \varphi\right)\left(x^{\prime}\right) & =\frac{1}{\sqrt{2 \pi \hbar}} \int \tilde{\varphi}(p) \sqrt{\left|\frac{\partial^{2} F_{4}}{\partial x^{\prime} \partial p}\left(x^{\prime}, p\right)\right|} e^{\frac{i}{\hbar} F_{4}\left(x^{\prime}, p\right)} \mathrm{d} p=\frac{1}{\sqrt{2 \pi \hbar}} \int \tilde{\varphi}(p)\left|\phi_{1}^{\prime}\left(x^{\prime}\right)\right|^{1 / 2} e^{-\frac{i}{\hbar}\left(p \phi_{1}\left(x^{\prime}\right)+\phi_{2}\left(x^{\prime}\right)\right)} \mathrm{d} p \\
& =\left|\phi_{1}^{\prime}\left(x^{\prime}\right)\right|^{1 / 2} e^{-\frac{i}{\hbar} \phi_{2}\left(x^{\prime}\right)} \varphi\left(\phi_{1}\left(x^{\prime}\right)\right)
\end{aligned}
$$

where $\tilde{\varphi}$ denotes the inverse Fourier transform of $\varphi$, indeed satisfies (15). The inverse operator to $\hat{U}_{T_{4}}$ is then given by

$$
\begin{aligned}
\left(\hat{U}_{T_{4}}^{-1} \varphi\right)(x) & =\frac{1}{\sqrt{2 \pi \hbar}} \mathcal{F}^{-1} \int \varphi\left(x^{\prime}\right) \sqrt{\left|\frac{\partial^{2} F_{4}}{\partial x^{\prime} \partial p}\left(x^{\prime}, p\right)\right|} e^{-\frac{i}{\hbar} F_{4}\left(x^{\prime}, p\right)} \mathrm{d} x^{\prime} \\
& =\frac{1}{2 \pi \hbar} \iint \varphi\left(x^{\prime}\right)\left|\phi_{1}^{\prime}\left(x^{\prime}\right)\right|^{1 / 2} e^{\frac{i}{\hbar}\left(p \phi_{1}\left(x^{\prime}\right)+\phi_{2}\left(x^{\prime}\right)-x p\right)} \mathrm{d} x^{\prime} \mathrm{d} p \\
& =\left|\phi_{1}^{\prime}\left(\phi_{1}^{-1}(x)\right)\right|^{-1 / 2} e^{\frac{i}{\hbar} \phi_{2}\left(\phi_{1}^{-1}(x)\right)} \varphi\left(\phi_{1}^{-1}(x)\right) .
\end{aligned}
$$

One can show that, in accordance with (12c), the operator $\hat{U}_{T_{4}}$ can be constructed from the operator $\hat{U}_{T_{1}}$ from (16) and the Fourier transform

$$
\hat{U}_{T_{4}}=\hat{U}_{I^{-1} \circ T_{1}}=\hat{U}_{T_{1}} \hat{U}_{I}^{-1}=\hat{U}_{T_{1}} \mathcal{F}^{-1},
$$


because $\hat{U}_{I}$ is exactly the Fourier transform $\mathcal{F}$. In analogical way are constructed unitary operators $\hat{U}_{T_{2}}$ and $\hat{U}_{T_{3}}$ :

$$
\begin{aligned}
& \hat{U}_{T_{2}}=\hat{U}_{I \circ T_{1} \circ I^{-1}}=\hat{U}_{I}^{-1} \hat{U}_{T_{1}} \hat{U}_{I}=\mathcal{F}^{-1} \hat{U}_{T_{1}} \mathcal{F}, \\
& \hat{U}_{T_{3}}=\hat{U}_{T_{1} \circ I^{-1}}=\hat{U}_{I}^{-1} \hat{U}_{T_{1}}=\mathcal{F}^{-1} \hat{U}_{T_{1}} .
\end{aligned}
$$

For a $2 N$-dimensional case and the transformation $T$ given by the generating function (13), the respective operator $\hat{U}_{T}$ takes the form

$$
\begin{aligned}
\left(\hat{U}_{T} \varphi\right)\left(x^{\prime}\right) & =\frac{1}{(2 \pi \hbar)^{N / 2}} \int \varphi(x)\left|\operatorname{det} \phi_{1}^{\prime}\left(x^{\prime}\right)\right|^{1 / 2} e^{-\frac{i}{\hbar}\left(\sum_{i} x^{i} \phi_{1}^{i}\left(x^{\prime}\right)+\phi_{2}\left(x^{\prime}\right)\right)} \mathrm{d} x \\
& =\frac{1}{(2 \pi \hbar)^{N / 2}} \int \varphi(x) \sqrt{\left|\operatorname{det}\left[\frac{\partial^{2} F}{\partial x^{i} \partial x^{\prime j}}\left(x, x^{\prime}\right)\right]\right|} e^{-\frac{i}{\hbar} F\left(x, x^{\prime}\right)} \mathrm{d} x .
\end{aligned}
$$

\section{Examples}

\subsection{Linear transformations}

In what follows the well known linear transformations of coordinates of a quantum phase space will be reconsidered in the frame of formalism developed in previous sections. The linear transformation is a transformation $T: \mathbb{R}^{2} \rightarrow \mathbb{R}^{2}$ given by the equation

$$
T\left(x^{\prime}, p^{\prime}\right)=\left(d x^{\prime}-b p^{\prime},-c x^{\prime}+a p^{\prime}\right),
$$

where $a, b, c, d \in \mathbb{R}$. Moreover, it is assumed that $a d-b c=1$, which makes this transformation canonical both in a classical and quantum phase space, i.e., it preserves both Poisson bracket and star-commutator. The inverse transformation is given by the following equation

$$
T^{-1}(x, p)=(a x+b p, c x+d p) .
$$

The linear transformation $T$ is generated by a function $F\left(x, x^{\prime}\right)=\frac{1}{b} x x^{\prime}-\frac{a}{2 b} x^{2}-\frac{d}{2 b} x^{2}$, i.e.,

$$
p=\frac{\partial F}{\partial x}\left(x, x^{\prime}\right), \quad p^{\prime}=-\frac{\partial F}{\partial x^{\prime}}\left(x, x^{\prime}\right)
$$

where $(x, p)=T\left(x^{\prime}, p^{\prime}\right)$.

For a given function $f \in C^{\infty}\left(\mathbb{R}^{2}\right)$ the derivatives of the function $f$ transform as follows

$$
\begin{aligned}
& \frac{\partial f}{\partial x} \circ T=a \frac{\partial}{\partial x^{\prime}}(f \circ T)+c \frac{\partial}{\partial p^{\prime}}(f \circ T), \\
& \frac{\partial f}{\partial p} \circ T=b \frac{\partial}{\partial x^{\prime}}(f \circ T)+d \frac{\partial}{\partial p^{\prime}}(f \circ T) .
\end{aligned}
$$

Using the above formulae one easily finds that the linear transformation $T$ preserves the $\star$-product, i.e., the $\star$-product do not change after the transformation of coordinates

$$
(f \star g) \circ T=(f \circ T) \star^{\prime}(g \circ T), \quad f, g \in C^{\infty}\left(\mathbb{R}^{2}\right) .
$$

Of course, in this case the isomorphism $S_{T}=1$.

Now, let us prove by direct calculation that the unitary operator $\hat{U}_{T}: L^{2}(\mathbb{R}) \rightarrow L^{2}(\mathbb{R})$ of the Hilbert space $L^{2}(\mathbb{R})$ induced by $T$ indeed is of the form (18). In other words, let us show that $\hat{U}_{T}$ given by (18) satisfies the formula

$$
\hat{T}\left(\varphi^{*} \otimes_{M} \psi\right)=\left(\varphi^{*} \otimes_{M} \psi\right) \circ T=\left(\hat{U}_{T} \varphi\right)^{*} \otimes_{M} \hat{U}_{T} \psi,
$$

for every $\varphi, \psi \in L^{2}(\mathbb{R})$. 
Indeed, the twisted tensor product of $\varphi^{*}$ and $\psi$ takes the form

$$
\left(\varphi^{*} \otimes_{M} \psi\right)(x, p)=\frac{1}{\sqrt{2 \pi \hbar}} \int \mathrm{d} y \varphi^{*}\left(x-\frac{1}{2} y\right) \psi\left(x+\frac{1}{2} y\right) e^{-\frac{i}{\hbar} p y} .
$$

Using the above formula the left side of (21) takes the form

$$
\begin{aligned}
\hat{T}\left(\varphi^{*} \otimes_{M} \psi\right)\left(x^{\prime}, p^{\prime}\right) & =\left(\varphi^{*} \otimes_{M} \psi\right)\left(T\left(x^{\prime}, p^{\prime}\right)\right) \\
& =\frac{1}{\sqrt{2 \pi \hbar}} \int \mathrm{d} y \varphi^{*}\left(d x^{\prime}-b p^{\prime}-\frac{1}{2} y\right) \psi\left(d x^{\prime}-b p^{\prime}+\frac{1}{2} y\right) e^{-\frac{i}{\hbar}\left(-c x^{\prime}+a p^{\prime}\right) y} .
\end{aligned}
$$

The right side of (21) takes the form

$$
\begin{aligned}
\left(\left(\hat{U}_{T} \varphi\right)^{*} \otimes_{M} \hat{U}_{T} \psi\right)\left(x^{\prime}, p^{\prime}\right)= & \frac{1}{\sqrt{2 \pi \hbar}} \int \mathrm{d} y^{\prime}\left(\hat{U}_{T} \varphi\right)^{*}\left(x^{\prime}-\frac{1}{2} y^{\prime}\right)\left(\hat{U}_{T} \psi\right)\left(x^{\prime}+\frac{1}{2} y^{\prime}\right) e^{-\frac{i}{\hbar} p^{\prime} y^{\prime}} \\
= & \frac{1}{(2 \pi \hbar)^{3 / 2}|b|} \iiint \mathrm{d} x \mathrm{~d} y \mathrm{~d} y^{\prime} \varphi^{*}(x) \psi(y) e^{\frac{i}{\hbar} F\left(x, x^{\prime}-\frac{1}{2} y^{\prime}\right)} e^{-\frac{i}{\hbar} F\left(y, x^{\prime}+\frac{1}{2} y^{\prime}\right)} e^{-\frac{i}{\hbar} p^{\prime} y^{\prime}} \\
= & \frac{1}{(2 \pi \hbar)^{3 / 2}|b|} \iint \mathrm{d} x \mathrm{~d} y \varphi^{*}(x) \psi(y) e^{-\frac{i}{\hbar}\left(\frac{1}{b}(y-x) x^{\prime}-\frac{a}{2 b}\left(y^{2}-x^{2}\right)\right)} \\
& \times \int \mathrm{d} y^{\prime} e^{-\frac{i}{\hbar}\left(\frac{1}{2 b}(x+y)-\frac{d}{b} x^{\prime}+p^{\prime}\right) y^{\prime}}
\end{aligned}
$$

After changing the variables under the integral sign from $(x, y)$ to $\left(x-\frac{1}{2} y, x+\frac{1}{2} y\right)$ and from $y^{\prime}$ to $b y^{\prime}$ the above equation takes the form

$$
\begin{aligned}
\left(\left(\hat{U}_{T} \varphi\right)^{*} \otimes_{M} \hat{U}_{T} \psi\right)\left(x^{\prime}, p^{\prime}\right)= & \frac{1}{(2 \pi \hbar)^{3 / 2}} \iint \mathrm{d} x \mathrm{~d} y \varphi^{*}\left(x-\frac{1}{2} y\right) \psi\left(x+\frac{1}{2} y\right) e^{-\frac{i}{\hbar}\left(\frac{1}{b} y x^{\prime}-\frac{a}{b} x y\right)} \\
& \times \int \mathrm{d} y^{\prime} e^{-\frac{i}{\hbar}\left(x-d x^{\prime}+b p^{\prime}\right) y^{\prime}} \\
= & \frac{1}{\sqrt{2 \pi \hbar}} \iint \mathrm{d} x \mathrm{~d} y \varphi^{*}\left(x-\frac{1}{2} y\right) \psi\left(x+\frac{1}{2} y\right) e^{-\frac{i}{\hbar}\left(\frac{1}{b} y x^{\prime}-\frac{a}{b} x y\right)} \delta\left(x-d x^{\prime}+b p^{\prime}\right) \\
= & \frac{1}{\sqrt{2 \pi \hbar}} \int \mathrm{d} y \varphi^{*}\left(d x^{\prime}-b p^{\prime}-\frac{1}{2} y\right) \psi\left(d x^{\prime}-b p^{\prime}+\frac{1}{2} y\right) e^{-\frac{i}{\hbar}\left(-c x^{\prime}+a p^{\prime}\right) y}
\end{aligned}
$$

Linear transformations of coordinates appear when considering quantum trajectories generated by Hamiltonian functions, quadratic with respect to canonical coordinates. Take as an example the Hamiltonian of harmonic oscillator

$$
H(x, p)=\frac{1}{2}\left(p^{2}+\omega^{2} x^{2}\right)
$$

It happens that in such case the quantum trajectory coincides with the classical one. Thus a classical/quantum trajectory $\Phi_{t}=(Q(t), P(t))$ of a harmonic oscillator takes the form

$$
Q(x, p, t)=x \cos \omega t+\omega^{-1} p \sin \omega t, \quad P(x, p, t)=p \cos \omega t-\omega x \sin \omega t .
$$

One can immediately see that for every $t$ the above equations define a linear canonical transformation with generating function

$$
F\left(x, x^{\prime}\right)=\frac{\omega}{\sin \omega t} x x^{\prime}-\frac{1}{2} \omega \cot \omega t x^{2}-\frac{1}{2} \omega \cot \omega t x^{\prime 2} .
$$

Concluding, the above results show that applying the Born's quantization rule to any classical observable transformed by canonical linear coordinate transformation, gives an operator unitary equivalent to an operator corresponding to untransformed classical observable, with the same ordering of $\hat{q}^{\prime}$ and $\hat{p}^{\prime}$. 


\subsection{A class of nonlinear transformations}

In the following example a class of nonlinear transformations of coordinates of a quantum phase space will be considered. The transformation in question will be taken in the form

$$
T\left(x^{\prime}, p^{\prime}\right)=\left(-a p^{\prime}-a \phi^{\prime}\left(x^{\prime}\right), a^{-1} x^{\prime}\right) .
$$

The transformation $T$ is generated by the function $F\left(x, x^{\prime}\right)=a^{-1} x x^{\prime}+\phi\left(x^{\prime}\right)(a \in \mathbb{R}, a \neq 0, \phi$ being an arbitrary smooth function). This transformation is a classical canonical transformation. For a given function $f \in C^{\infty}\left(\mathbb{R}^{2}\right)$ the derivatives of the function $f$ transform as follows

$$
\begin{aligned}
& \frac{\partial f}{\partial x} \circ T=-\frac{1}{a} \frac{\partial}{\partial p^{\prime}}(f \circ T), \\
& \frac{\partial f}{\partial p} \circ T=a \frac{\partial}{\partial x^{\prime}}(f \circ T)-a \phi^{\prime \prime}\left(x^{\prime}\right) \frac{\partial}{\partial p^{\prime}}(f \circ T) .
\end{aligned}
$$

Hence the Moyal $\star$-product transforms to the following product

$$
f \star_{T}^{\prime} g=f \exp \left(\frac{1}{2} i \hbar \overleftarrow{D_{x^{\prime}}} \overrightarrow{D_{p^{\prime}}}-\frac{1}{2} i \hbar \overleftarrow{D_{p^{\prime}}} \overrightarrow{D_{x^{\prime}}}\right) g
$$

where

$$
\begin{aligned}
& D_{x^{\prime}}=a^{-1} \partial_{p^{\prime}}, \\
& D_{p^{\prime}}=a \partial_{x^{\prime}}-a \phi^{\prime \prime}\left(x^{\prime}\right) \partial_{p^{\prime}} .
\end{aligned}
$$

One can calculate that

$$
\begin{aligned}
& \hat{q}_{T}^{\prime}=x^{\prime} \star_{T}^{\prime}=x^{\prime}+\frac{1}{2} i \hbar \partial_{p^{\prime}} \\
& \hat{p}_{T}^{\prime}=p^{\prime} \star_{T}^{\prime}=p^{\prime}-\frac{1}{2} i \hbar \partial_{x^{\prime}}-\sum_{n=2}^{\infty} \frac{1}{n !}\left(\frac{i \hbar}{2}\right)^{n} \phi^{(n+1)}\left(x^{\prime}\right) \partial_{p^{\prime}}^{n}
\end{aligned}
$$

and that

$$
\left[\hat{q}_{T}^{\prime}, \hat{p}_{T}^{\prime}\right]=i \hbar .
$$

The above equation shows that the transformation $T$ is a quantum canonical transformation.

The isomorphism $S_{T}$ from (6) intertwining the Moyal $\star^{\prime}$-product with the $\star_{T}^{\prime}$-product is given by

$$
\begin{aligned}
S_{T} & =\exp \left(-\sum_{n=1}^{\infty} \frac{1}{(2 n+1) !}(-1)^{n}\left(\frac{\hbar}{2}\right)^{2 n} \phi^{(2 n+1)}\left(x^{\prime}\right) \partial_{p^{\prime}}^{2 n+1}\right) \\
& =\exp \left(\frac{i}{\hbar}\left(\phi\left(x^{\prime}+\frac{1}{2} i \hbar \partial_{p^{\prime}}\right)-\phi\left(x^{\prime}-\frac{1}{2} i \hbar \partial_{p^{\prime}}\right)-i \hbar \phi^{\prime}\left(x^{\prime}\right) \partial_{p^{\prime}}\right)\right) \\
& =\exp \left(\frac{i}{\hbar}\left(\phi\left(\hat{q}_{M}\right)-\phi^{\prime}\left(x^{\prime}\right) \hat{q}_{M}-\phi\left(\hat{q}_{M}^{*}\right)+\phi^{\prime}\left(x^{\prime}\right) \hat{q}_{M}^{*}\right)\right)
\end{aligned}
$$

Indeed, from Theorem 3.1 it is enough to prove that

$$
\begin{aligned}
& \hat{q}_{T}^{\prime}=S_{T} \hat{q}_{M} S_{T}^{-1}, \\
& \hat{p}_{T}^{\prime}=S_{T} \hat{p}_{M} S_{T}^{-1} .
\end{aligned}
$$

Since $S_{T}=e^{\hat{A}}$ where $\hat{A}=-\sum_{n=1}^{\infty} \frac{1}{(2 n+1) !}(-1)^{n}\left(\frac{\hbar}{2}\right)^{2 n} \phi^{(2 n+1)}\left(x^{\prime}\right) \partial_{p^{\prime}}^{2 n+1}$ the above equations, from the Hadamard's lemma, take the form

$$
\begin{aligned}
& \hat{q}_{T}^{\prime}=e^{[\hat{A}, \cdot]} \hat{q}_{M}, \\
& \hat{p}_{T}^{\prime}=e^{[\hat{A}, \cdot]} \hat{p}_{M} .
\end{aligned}
$$


One easily calculates that $\left[\hat{A}, \hat{q}_{M}\right]=0$. Thus

$$
e^{[\hat{A}, \cdot]} \hat{q}_{M}=\hat{q}_{M}=\hat{q}_{T}^{\prime},
$$

which proves (22a).

On the other hand, one finds that

$$
\begin{aligned}
{\left[\hat{A}, \hat{p}_{M}\right] } & =-\sum_{n=1}^{\infty} \frac{1}{(2 n) !}(-1)^{n}\left(\frac{\hbar}{2}\right)^{2 n} \phi^{(2 n+1)}\left(x^{\prime}\right) \partial_{p^{\prime}}^{2 n}-\frac{1}{2} i \hbar \sum_{n=1}^{\infty} \frac{1}{(2 n+1) !}(-1)^{n}\left(\frac{\hbar}{2}\right)^{2 n} \phi^{(2 n+2)}\left(x^{\prime}\right) \partial_{p^{\prime}}^{2 n+1} \\
& =-\sum_{n=2}^{\infty} \frac{1}{n !}\left(\frac{i \hbar}{2}\right)^{n} \phi^{(n+1)}\left(x^{\prime}\right) \partial_{p^{\prime}}^{n}
\end{aligned}
$$

and that

$$
\left[\hat{A},\left[\hat{A}, \hat{p}_{M}\right]\right]=0
$$

Thus

$$
e^{[\hat{A}, \cdot]} \hat{p}_{M}=p^{\prime}-\frac{1}{2} i \hbar \partial_{x^{\prime}}-\sum_{n=2}^{\infty} \frac{1}{n !}\left(\frac{i \hbar}{2}\right)^{n} \phi^{(n+1)}\left(x^{\prime}\right) \partial_{p^{\prime}}^{n}=\hat{p}_{T}^{\prime}
$$

which proves (22b).

To derive the second formula on $S_{T}$ from the first one note that

$$
\begin{aligned}
S_{T} & =\exp \left(-\sum_{n=1}^{\infty} \frac{1}{(n+1) !}\left(\frac{i \hbar}{2}\right)^{n} \frac{1+(-1)^{n}}{2} \phi^{(n+1)}\left(x^{\prime}\right) \partial_{p^{\prime}}^{n+1}\right) \\
& =\exp \left(\frac{i}{\hbar} \sum_{n=2}^{\infty} \frac{1}{n !}\left(1-(-1)^{n}\right) \phi^{(n)}\left(x^{\prime}\right)\left(\frac{1}{2} i \hbar \partial_{p^{\prime}}\right)^{n}\right) \\
& =\exp \left(\frac{i}{\hbar}\left(\phi\left(x^{\prime}+\frac{1}{2} i \hbar \partial_{p^{\prime}}\right)-\phi\left(x^{\prime}\right)-\phi^{\prime}\left(x^{\prime}\right) \frac{1}{2} i \hbar \partial_{p^{\prime}}-\phi\left(x^{\prime}-\frac{1}{2} i \hbar \partial_{p^{\prime}}\right)+\phi\left(x^{\prime}\right)-\phi^{\prime}\left(x^{\prime}\right) \frac{1}{2} i \hbar \partial_{p^{\prime}}\right)\right) \\
& =\exp \left(\frac{i}{\hbar}\left(\phi\left(x^{\prime}+\frac{1}{2} i \hbar \partial_{p^{\prime}}\right)-\phi\left(x^{\prime}-\frac{1}{2} i \hbar \partial_{p^{\prime}}\right)-i \hbar \phi^{\prime}\left(x^{\prime}\right) \partial_{p^{\prime}}\right)\right) .
\end{aligned}
$$

Observe, that the map $S_{T}$ preserves the involution, i.e.,

$$
S_{T}\left(f^{*}\right)=\left(S_{T} f\right)^{*}, \quad f \in \mathcal{A}_{Q},
$$

which can be immediately seen from the fact that $S_{T}=S_{T}^{*}$.

In accordance with the assumption made in Subsection 3.1 concerning $S_{T}$ the map $S_{T}$ is an isomorphism (unitary operator) of the space of states $\mathcal{H}=L^{2}\left(\mathbb{R}^{2}\right)$ onto the transformed space of states $\mathcal{H}^{\prime}=L^{2}\left(\mathbb{R}^{2}\right)$.

Indeed, an operator of the form $\exp \left(\frac{i}{\hbar} f\left(x^{\prime}, i \hbar \partial_{p^{\prime}}\right)\right)\left(f: \mathbb{R}^{2} \rightarrow \mathbb{R}\right.$ being some smooth function) transforms square integrable functions into square integrable functions. In fact, this operator can be written in the following form

$$
\exp \left(\frac{i}{\hbar} f\left(x^{\prime}, i \hbar \partial_{p^{\prime}}\right)\right)=\mathcal{F}_{p}^{-1} \exp \left(\frac{i}{\hbar} f\left(x^{\prime}, \eta\right)\right) \mathcal{F}_{p}
$$

as a composition of operators transforming square integrable functions into square integrable functions. Hence $S_{T}: L^{2}\left(\mathbb{R}^{2}\right) \rightarrow L^{2}\left(\mathbb{R}^{2}\right)$. The map $S_{T}$ is obviously linear and injective, moreover, it is easy to show that $\left\langle\Phi \mid S_{T} \Psi\right\rangle_{L^{2}}=\left\langle S_{T}^{-1} \Phi \mid \Psi\right\rangle_{L^{2}}$, thus $S_{T}$ is an isomorphism of $L^{2}\left(\mathbb{R}^{2}\right)$ onto $L^{2}\left(\mathbb{R}^{2}\right)$.

Now, let us prove by direct calculation that the unitary operator $\hat{U}_{T}: L^{2}(\mathbb{R}) \rightarrow L^{2}(\mathbb{R})$ of the Hilbert space $L^{2}(\mathbb{R})$ induced by $T$ indeed is of the form (18). In other words, let us show that $\hat{U}_{T}$ given by (18) satisfies the formula

$$
\hat{T}\left(\varphi^{*} \otimes_{M} \psi\right)=\left(\varphi^{*} \otimes_{M} \psi\right) \circ T=\left(\hat{U}_{T} \varphi\right)^{*} \otimes_{S_{T}} \hat{U}_{T} \psi,
$$


for every $\varphi, \psi \in L^{2}(\mathbb{R})$.

Indeed, the left side of (23) takes the form

$$
\left(\varphi^{*} \otimes_{M} \psi\right)\left(T\left(x^{\prime}, p^{\prime}\right)\right)=\frac{1}{\sqrt{2 \pi \hbar}} \int \mathrm{d} y^{\prime} \varphi^{*}\left(-a p^{\prime}-a \phi^{\prime}\left(x^{\prime}\right)-\frac{1}{2} y^{\prime}\right) \psi\left(-a p^{\prime}-a \phi^{\prime}\left(x^{\prime}\right)+\frac{1}{2} y^{\prime}\right) e^{-\frac{i}{\hbar} a^{-1} x^{\prime} y^{\prime}} .
$$

The right side of (23) takes the form

$$
\begin{aligned}
\left(\left(\hat{U}_{T} \varphi\right)^{*} \otimes_{S_{T}} \hat{U}_{T} \psi\right)\left(x^{\prime}, p^{\prime}\right)= & S_{T} \frac{1}{\sqrt{2 \pi \hbar}} \int \mathrm{d} y^{\prime \prime}\left(\hat{U}_{T} \varphi\right)^{*}\left(x^{\prime}-\frac{1}{2} y^{\prime \prime}\right)\left(\hat{U}_{T} \psi\right)\left(x^{\prime}+\frac{1}{2} y^{\prime \prime}\right) e^{-\frac{i}{\hbar} p^{\prime} y^{\prime \prime}} \\
= & S_{T} \frac{1}{(2 \pi \hbar)^{3 / 2}}|a|^{-1} \iiint \mathrm{d} y^{\prime \prime} \mathrm{d} x \mathrm{~d} y \varphi^{*}(x) e^{\frac{i}{\hbar}\left(a^{-1} x\left(x^{\prime}-\frac{1}{2} y^{\prime \prime}\right)+\phi\left(x^{\prime}-\frac{1}{2} y^{\prime \prime}\right)\right)} \\
& \times \psi(y) e^{-\frac{i}{\hbar}\left(a^{-1} y\left(x^{\prime}+\frac{1}{2} y^{\prime \prime}\right)+\phi\left(x^{\prime}+\frac{1}{2} y^{\prime \prime}\right)\right)} e^{-\frac{i}{\hbar} p^{\prime} y^{\prime \prime}} .
\end{aligned}
$$

Since

$$
\begin{gathered}
\exp \left(\frac{i}{\hbar}\left(\phi\left(x^{\prime}+\frac{1}{2} i \hbar \partial_{p^{\prime}}\right)-\phi\left(x^{\prime}-\frac{1}{2} i \hbar \partial_{p^{\prime}}\right)-i \hbar \phi^{\prime}\left(x^{\prime}\right) \partial_{p^{\prime}}\right)\right) g\left(x^{\prime}\right) e^{-\frac{i}{\hbar} p^{\prime} y^{\prime \prime}}= \\
\quad=\exp \left(\frac{i}{\hbar}\left(\phi\left(x^{\prime}+\frac{1}{2} y^{\prime \prime}\right)-\phi\left(x^{\prime}-\frac{1}{2} y^{\prime \prime}\right)-\phi^{\prime}\left(x^{\prime}\right) y^{\prime \prime}\right)\right) g\left(x^{\prime}\right) e^{-\frac{i}{\hbar} p^{\prime} y^{\prime \prime}}
\end{gathered}
$$

(24) takes the form

$$
\begin{aligned}
& \left(\left(\hat{U}_{T} \varphi\right)^{*} \otimes_{S_{T}} \hat{U}_{T} \psi\right)\left(x^{\prime}, p^{\prime}\right)=\frac{1}{(2 \pi \hbar)^{3 / 2}}|a|^{-1} \iiint \mathrm{d} y^{\prime \prime} \mathrm{d} x \mathrm{~d} y \varphi^{*}(x) \psi(y) \\
& \quad \times \exp \left(\frac { i } { \hbar } \left(a^{-1}(x-y) x^{\prime}-a^{-1}\left(\frac{1}{2} x+\frac{1}{2} y\right) y^{\prime \prime}+\phi\left(x^{\prime}-\frac{1}{2} y^{\prime \prime}\right)-\phi\left(x^{\prime}+\frac{1}{2} y^{\prime \prime}\right)\right.\right. \\
& \left.\left.\quad-\phi\left(x^{\prime}-\frac{1}{2} y^{\prime \prime}\right)+\phi\left(x^{\prime}+\frac{1}{2} y^{\prime \prime}\right)-\phi^{\prime}\left(x^{\prime}\right) y^{\prime \prime}-p^{\prime} y^{\prime \prime}\right)\right) \\
& =\frac{1}{(2 \pi \hbar)^{3 / 2}}|a|^{-1} \iiint \mathrm{d} y^{\prime \prime} \mathrm{d} x \mathrm{~d} y \varphi^{*}(x) \psi(y) \\
& \quad \times \exp \left(\frac{i}{\hbar}\left(-a^{-1}(y-x) x^{\prime}-\left(a^{-1}\left(\frac{1}{2} x+\frac{1}{2} y\right)+\phi^{\prime}\left(x^{\prime}\right)+p^{\prime}\right) y^{\prime \prime}\right)\right) .
\end{aligned}
$$

Introducing new variables $y^{\prime}=y-x$ and $z^{\prime}=a^{-1}\left(\frac{1}{2} x+\frac{1}{2} y\right)+\phi^{\prime}\left(x^{\prime}\right)+p^{\prime}$ the above equation can be written in the form

$$
\begin{aligned}
& \left(\left(\hat{U}_{T} \varphi\right)^{*} \otimes_{S_{T}} \hat{U}_{T} \psi\right)\left(x^{\prime}, p^{\prime}\right)=\frac{1}{(2 \pi \hbar)^{3 / 2}} \iiint \mathrm{d} y^{\prime \prime} \mathrm{d} y^{\prime} \mathrm{d} z^{\prime} \varphi^{*}\left(a z^{\prime}-a \phi^{\prime}\left(x^{\prime}\right)-a p^{\prime}-\frac{1}{2} y^{\prime}\right) \\
& \times \psi\left(a z^{\prime}-a \phi^{\prime}\left(x^{\prime}\right)-a p^{\prime}+\frac{1}{2} y^{\prime}\right) e^{-\frac{i}{\hbar} a x^{\prime} y^{\prime}} e^{-\frac{i}{\hbar} z^{\prime} y^{\prime \prime}} \\
& =\frac{1}{\sqrt{2 \pi \hbar}} \int \mathrm{d} y^{\prime} \varphi^{*}\left(-a p^{\prime}-a \phi^{\prime}\left(x^{\prime}\right)-\frac{1}{2} y^{\prime}\right) \psi\left(-a p^{\prime}-a \phi^{\prime}\left(x^{\prime}\right)+\frac{1}{2} y^{\prime}\right) e^{-\frac{i}{\hbar} a^{-1} x^{\prime} y^{\prime}}
\end{aligned}
$$

\subsection{Nonlinear point transformations}

Let us consider in the following example the point transformations of phase space coordinates

$$
T\left(x^{\prime}, p^{\prime}\right)=\left(\phi\left(x^{\prime}\right),\left(\phi^{\prime}\left(x^{\prime}\right)\right)^{-1} p^{\prime}\right)
$$

generated by functions $F\left(x^{\prime}, p\right)=-p \phi\left(x^{\prime}\right)$ ( $\phi$ being an arbitrary smooth bijective function). These transformations from construction are classical canonical transformations. For a given function $f \in C^{\infty}\left(\mathbb{R}^{2}\right)$ the 
derivatives of the function $f$ transform as follows

$$
\begin{aligned}
& \frac{\partial f}{\partial x} \circ T=\left(\phi^{\prime}\left(x^{\prime}\right)\right)^{-2} \phi^{\prime \prime}\left(x^{\prime}\right) p^{\prime} \frac{\partial}{\partial p^{\prime}}(f \circ T)+\left(\phi^{\prime}\left(x^{\prime}\right)\right)^{-1} \frac{\partial}{\partial x^{\prime}}(f \circ T), \\
& \frac{\partial f}{\partial p} \circ T=\phi^{\prime}\left(x^{\prime}\right) \frac{\partial}{\partial p^{\prime}}(f \circ T) .
\end{aligned}
$$

Hence the Moyal $\star$-product transforms to the following product

$$
f \star_{T}^{\prime} g=f \exp \left(\frac{1}{2} i \hbar \overleftarrow{D_{x^{\prime}}} \overrightarrow{D_{p^{\prime}}}-\frac{1}{2} i \hbar \overleftarrow{D_{p^{\prime}}} \overrightarrow{D_{x^{\prime}}}\right) g
$$

where

$$
\begin{aligned}
& D_{x^{\prime}}=\left(\phi^{\prime}\left(x^{\prime}\right)\right)^{-1} \partial_{x^{\prime}}+\left(\phi^{\prime}\left(x^{\prime}\right)\right)^{-2} \phi^{\prime \prime}\left(x^{\prime}\right) p^{\prime} \partial_{p^{\prime}} \\
& D_{p^{\prime}}=\phi^{\prime}\left(x^{\prime}\right) \partial_{p^{\prime}} .
\end{aligned}
$$

One can calculate that

$$
\begin{aligned}
\hat{q}_{T}^{\prime}= & x^{\prime} \star_{T}^{\prime}=x^{\prime}+\frac{1}{2} i \hbar \partial_{p^{\prime}} \\
& +\sum_{n=1}^{\infty} \frac{1}{(n+1) !}\left(\frac{i \hbar}{2}\right)^{n+1}\left(\phi^{\prime}\left(x^{\prime}\right)\right)^{-n} \sum_{\left(k_{1}, \ldots, k_{n}\right) \in G_{n}} a_{k_{1}, \ldots, k_{n}}^{(n)} \phi^{\left(k_{1}\right)}\left(x^{\prime}\right) \cdots \phi^{\left(k_{n}\right)}\left(x^{\prime}\right) \partial_{x^{\prime}} \partial_{p^{\prime}}^{n+1}, \\
\hat{p}_{T}^{\prime}= & p^{\prime} \star_{T}^{\prime}=p^{\prime}-\frac{1}{2} i \hbar \partial_{x^{\prime}} \\
& +\sum_{n=1}^{\infty} \frac{1}{(n+1) !}\left(\frac{i \hbar}{2}\right)^{n+1}\left(\phi^{\prime}\left(x^{\prime}\right)\right)^{-n-1}\left(\sum_{\left(k_{1}, \ldots, k_{n+1}\right) \in G_{n+1}} b_{k_{1}, \ldots, k_{n+1}}^{(n+1)} \phi^{\left(k_{1}\right)}\left(x^{\prime}\right) \cdots \phi^{\left(k_{n+1}\right)}\left(x^{\prime}\right) p^{\prime} \partial_{p^{\prime}}^{n+1}\right. \\
& +\sum_{\left(k_{1}, \ldots, k_{n}\right) \in G_{n}} c_{k_{1}, \ldots, k_{n}}^{(n)} \phi^{\left(k_{1}\right)}\left(x^{\prime}\right) \cdots \phi^{\left(k_{n}\right)}\left(x^{\prime}\right) \phi^{\prime}\left(x^{\prime}\right) \partial_{x^{\prime}} \partial_{p^{\prime}}^{n} \\
& \left.+\sum_{\left(k_{1}, \ldots, k_{n}\right) \in G_{n}} n c_{k_{1}, \ldots, k_{n}}^{(n)} \phi^{\left(k_{1}\right)}\left(x^{\prime}\right) \cdots \phi^{\left(k_{n}\right)}\left(x^{\prime}\right) \phi^{\prime \prime}\left(x^{\prime}\right) \partial_{p^{\prime}}^{n}\right)
\end{aligned}
$$

where $G_{n}=\left\{\left(k_{1}, \ldots, k_{n}\right) \in \mathbb{N}^{n}: 1 \leq k_{1} \leq \ldots \leq k_{n}, \sum_{i=1}^{n} k_{i}=2 n\right\}$ and $a_{k_{1}, \ldots, k_{n}}^{(n)}, b_{k_{1}, \ldots, k_{n}}^{(n)}, c_{k_{1}, \ldots, k_{n}}^{(n)}$ are some integer constants. To the third order in $\hbar$ the operators $\hat{q}_{T}^{\prime}$ and $\hat{p}_{T}^{\prime}$ take the form

$$
\begin{aligned}
\hat{q}_{T}^{\prime}= & x^{\prime}+\frac{1}{2} i \hbar \partial_{p^{\prime}}+\frac{1}{2 !}\left(\frac{i \hbar}{2}\right)^{2}\left(\phi^{\prime}\left(x^{\prime}\right)\right)^{-1}\left(-\phi^{\prime \prime}\left(x^{\prime}\right)\right) \partial_{p^{\prime}}^{2} \\
& +\frac{1}{3 !}\left(\frac{i \hbar}{2}\right)^{3}\left(\phi^{\prime}\left(x^{\prime}\right)\right)^{-2}\left(3\left(\phi^{\prime \prime}\left(x^{\prime}\right)\right)^{2}-\phi^{\prime}\left(x^{\prime}\right) \phi^{\prime \prime \prime}\left(x^{\prime}\right)\right) \partial_{p^{\prime}}^{3}+o\left(\hbar^{4}\right) \\
\hat{p}_{T}^{\prime}= & p^{\prime}-\frac{1}{2} i \hbar \partial_{x^{\prime}}+\frac{1}{2 !}\left(\frac{i \hbar}{2}\right)^{2}\left(\phi^{\prime}\left(x^{\prime}\right)\right)^{-2}\left(\left(-3\left(\phi^{\prime \prime}\left(x^{\prime}\right)\right)^{2}+\phi^{\prime}\left(x^{\prime}\right) \phi^{\prime \prime \prime}\left(x^{\prime}\right)\right) p^{\prime} \partial_{p^{\prime}}^{2}\right. \\
& \left.+\left(-2 \phi^{\prime \prime}\left(x^{\prime}\right)\right) \phi^{\prime}\left(x^{\prime}\right) \partial_{x^{\prime}} \partial_{p^{\prime}}+\left(-2 \phi^{\prime \prime}\left(x^{\prime}\right)\right) \phi^{\prime \prime}\left(x^{\prime}\right) \partial_{p^{\prime}}\right) \\
& +\frac{1}{3 !}\left(\frac{i \hbar}{2}\right)^{3}\left(\phi^{\prime}\left(x^{\prime}\right)\right)^{-3}\left(\left(6\left(\phi^{\prime \prime}\left(x^{\prime}\right)\right)^{3}-7 \phi^{\prime}\left(x^{\prime}\right) \phi^{\prime \prime}\left(x^{\prime}\right) \phi^{\prime \prime \prime}\left(x^{\prime}\right)+\left(\phi^{\prime}\left(x^{\prime}\right)\right)^{2} \phi^{\prime \prime \prime \prime}\left(x^{\prime}\right)\right) p^{\prime} \partial_{p^{\prime}}^{3}\right. \\
& \left.+\left(3\left(\phi^{\prime \prime}\left(x^{\prime}\right)\right)^{2}-3 \phi^{\prime}\left(x^{\prime}\right) \phi^{\prime \prime \prime}\left(x^{\prime}\right)\right) \phi^{\prime}\left(x^{\prime}\right) \partial_{x^{\prime}} \partial_{p^{\prime}}^{2}+2\left(3\left(\phi^{\prime \prime}\left(x^{\prime}\right)\right)^{2}-3 \phi^{\prime}\left(x^{\prime}\right) \phi^{\prime \prime \prime}\left(x^{\prime}\right)\right) \phi^{\prime \prime}\left(x^{\prime}\right) \partial_{p^{\prime}}^{2}\right)+o\left(\hbar^{4}\right)
\end{aligned}
$$


Moreover, it can be verified that

$$
\left[\hat{q}_{T}^{\prime}, \hat{p}_{T}^{\prime}\right]=i \hbar
$$

which shows that $T$ is a quantum canonical transformation.

Using Theorem 3.1 it can be shown that the isomorphism $S_{T}$ is of the form

$$
\begin{aligned}
S_{T}= & \exp \left[\sum _ { n = 1 } ^ { \infty } \frac { 1 } { ( 2 n + 1 ) ! } ( \frac { \hbar } { 2 } ) ^ { 2 n } ( \phi ^ { \prime } ( x ^ { \prime } ) ) ^ { - 2 n } \left(\sum_{\left(k_{1}, \ldots, k_{2 n}\right) \in G_{2 n}} A_{k_{1}, \ldots, k_{2 n}}^{(2 n)} \phi^{\left(k_{1}\right)}\left(x^{\prime}\right) \cdots \phi^{\left(k_{2 n}\right)}\left(x^{\prime}\right) p^{\prime} \partial_{p^{\prime}}^{2 n+1}\right.\right. \\
& +\sum_{\left(k_{1}, \ldots, k_{2 n-1}\right) \in G_{2 n-1}} B_{k_{1}, \ldots, k_{2 n-1}}^{(2 n-1)} \phi^{\left(k_{1}\right)}\left(x^{\prime}\right) \cdots \phi^{\left(k_{2 n-1}\right)}\left(x^{\prime}\right) \phi^{\prime}\left(x^{\prime}\right) \partial_{x^{\prime}} \partial_{p^{\prime}}^{2 n} \\
& \left.\left.+\sum_{\left(k_{1}, \ldots, k_{2 n-1}\right) \in G_{2 n-1}} C_{k_{1}, \ldots, k_{2 n-1}}^{(2 n-1)} \phi^{\left(k_{1}\right)}\left(x^{\prime}\right) \cdots \phi^{\left(k_{2 n-1}\right)}\left(x^{\prime}\right) \phi^{\prime \prime}\left(x^{\prime}\right) \partial_{p^{\prime}}^{2 n}\right)\right]
\end{aligned}
$$

where $A_{k_{1}, \ldots, k_{n}}^{(n)}, B_{k_{1}, \ldots, k_{n}}^{(n)}, C_{k_{1}, \ldots, k_{n}}^{(n)}$ are some integer constants. To the second order in $\hbar$ the isomorphism $S_{T}$ reads

$$
\begin{aligned}
S_{T}= & 1+\frac{1}{3 !}\left(\frac{\hbar}{2}\right)^{2}\left(\phi^{\prime}\left(x^{\prime}\right)\right)^{-2}\left(\left(3\left(\phi^{\prime \prime}\left(x^{\prime}\right)\right)^{2}-\phi^{\prime}\left(x^{\prime}\right) \phi^{\prime \prime \prime}\left(x^{\prime}\right)\right) p^{\prime} \partial_{p^{\prime}}^{3}+\left(3 \phi^{\prime \prime}\left(x^{\prime}\right)\right) \phi^{\prime}\left(x^{\prime}\right) \partial_{x^{\prime}} \partial_{p^{\prime}}^{2}\right. \\
& \left.+\left(3 \phi^{\prime \prime}\left(x^{\prime}\right)\right) \phi^{\prime \prime}\left(x^{\prime}\right) \partial_{p^{\prime}}^{2}\right)+o\left(\hbar^{4}\right) .
\end{aligned}
$$

There is no simple expression for the constants $A_{k_{1}, \ldots, k_{n}}^{(n)}, B_{k_{1}, \ldots, k_{n}}^{(n)}, C_{k_{1}, \ldots, k_{n}}^{(n)}$ although, they can be calculated for particular cases of point transformations. One of such cases is for $\phi: \mathbb{R} \backslash\{0\} \rightarrow \mathbb{R} \backslash\{0\}$, $\phi\left(x^{\prime}\right)=\operatorname{sgn}\left(x^{\prime}\right) \sqrt{\left|2 x^{\prime}\right|}$ (the example from Introduction). The operators $\hat{q}_{T}^{\prime}$ and $\hat{p}_{T}^{\prime}$ take then the form

$$
\begin{aligned}
& \hat{q}_{T}^{\prime}=x^{\prime}+\frac{1}{2} i \hbar \partial_{p^{\prime}}-\frac{1}{8} \hbar^{2} \operatorname{sgn}\left(x^{\prime}\right)\left|2 x^{\prime}\right|^{-1} \partial_{p^{\prime}}^{2}, \\
& \hat{p}_{T}^{\prime}=p^{\prime}-\frac{1}{2} i \hbar \partial_{x^{\prime}}+\sum_{n=1}^{\infty}\left(-\frac{i \hbar}{2}\right)^{n+1}\left(\left(\operatorname{sgn}\left(x^{\prime}\right)\right)^{n}\left|2 x^{\prime}\right|^{-n} \partial_{x^{\prime}} \partial_{p^{\prime}}^{n}-n\left(\operatorname{sgn}\left(x^{\prime}\right)\right)^{n+1}\left|2 x^{\prime}\right|^{-n-1} \partial_{p^{\prime}}^{n}\right),
\end{aligned}
$$

and the isomorphism $S_{T}$ is expressed by the formula

$$
S_{T}=\exp \left(\sum_{n=1}^{\infty}(-1)^{n}\left(\frac{\hbar}{2}\right)^{2 n}\left(A_{n} \operatorname{sgn}\left(x^{\prime}\right)\left|2 x^{\prime}\right|^{-2 n+1} \partial_{x^{\prime}} \partial_{p^{\prime}}^{2 n}-B_{n}\left|2 x^{\prime}\right|^{-2 n} \partial_{p^{\prime}}^{2 n}\right)\right)
$$

where $A_{n}$ and $B_{n}$ are rational constants given recursively by

$$
\begin{aligned}
& A_{n}=\frac{1}{2 n}\left(1-\sum_{k=2}^{n} \frac{1}{k !} A_{2 n-1}^{(k)}\right), \\
& B_{n}=\frac{1}{2 n}\left(2 n-1-\sum_{k=2}^{n} \frac{1}{k !} B_{2 n-1}^{(k)}\right),
\end{aligned}
$$

where

$$
\begin{aligned}
& A_{2 n-1}^{(k)}=\sum_{m=1}^{n-1} 4(n-2 m) A_{n-m} A_{2 m-1}^{(k-1)} \\
& B_{2 n-1}^{(k)}=\sum_{m=1}^{n-1}\left(4(n-m) B_{n-m} A_{2 m-1}^{(k-1)}-4 m A_{n-m} B_{2 m-1}^{(k-1)}\right)
\end{aligned}
$$


for $k=2,3, \ldots, n$ and $n=2,3, \ldots$, and

$$
\begin{gathered}
A_{1}^{(k)}=B_{1}^{(k)}=0, \quad k=2,3, \ldots \\
A_{2 n-1}^{(1)}=2 n A_{n}, \quad B_{2 n-1}^{(1)}=2 n B_{n}, \quad n=1,2, \ldots .
\end{gathered}
$$

The values of couple first constants $A_{n}$ and $B_{n}$ are

$$
\begin{aligned}
& A_{1}=\frac{1}{2}, \\
& A_{2}=\frac{1}{4} \text {, } \\
& A_{3}=\frac{1}{4}, \\
& A_{4}=\frac{7}{24}, \\
& B_{1}=\frac{1}{2}, \\
& B_{2}=\frac{3}{4} \text {, } \\
& B_{3}=\frac{5}{4} \text {, } \\
& B_{4}=\frac{49}{24} \text {. }
\end{aligned}
$$

5.4. A nonlinear transformation in four-dimensions

In the following example we will demonstrate the presented theory of coordinate transformations on the nonlinear transformation $T\left(x^{\prime}, y^{\prime}, p_{1}^{\prime}, p_{2}^{\prime}\right)=\left(x, y, p_{1}, p_{2}\right)$ in four-dimensional phase space $\mathbb{R}^{4}$, where

$$
\begin{aligned}
x & =x^{\prime}-\frac{1}{m_{1}} t p_{1}^{\prime}-\frac{k}{2 m_{1}} t^{2} p_{2}^{\prime 2}, \\
y & =y^{\prime}-\frac{1}{m_{2}} t p_{2}^{\prime}-2 k t x^{\prime} p_{2}^{\prime}+\frac{k}{m_{1}} t^{2} p_{1}^{\prime} p_{2}^{\prime}+\frac{k^{2}}{3 m_{1}} t^{3} p_{2}^{\prime 3}, \\
p_{1} & =p_{1}^{\prime}+k t p_{2}^{\prime 2}, \\
p_{2} & =p_{2}^{\prime},
\end{aligned}
$$

and $m_{1}, m_{2}, k, t$ are some real constants. This transformation is a classical canonical transformation generated by a function

$$
F\left(x, y, p_{1}^{\prime}, p_{2}^{\prime}\right)=x p_{1}^{\prime}+y p_{2}^{\prime}+k t x p_{2}^{\prime 2}+\frac{1}{2 m_{1}} t p_{1}^{\prime 2}+\frac{1}{2 m_{2}} t p_{2}^{\prime 2}+\frac{k}{2 m_{1}} t^{2} p_{1}^{\prime} p_{2}^{\prime 2}+\frac{k^{2}}{6 m_{1}} t^{3} p_{2}^{\prime 4} .
$$

Note, that this transformation is a four-dimensional example of the transformation from (11a). It transforms the Moyal $\star$-product to the following product

$$
f \star_{T}^{\prime} g=f \exp \left(\frac{1}{2} i \hbar \overleftarrow{D_{x^{\prime}}} \overrightarrow{D_{p_{1}^{\prime}}}+\frac{1}{2} i \hbar \overleftarrow{D_{y^{\prime}}} \overrightarrow{D_{p_{2}^{\prime}}}-\frac{1}{2} i \hbar \overleftarrow{D_{p_{1}^{\prime}}} \overrightarrow{D_{x^{\prime}}}-\frac{1}{2} i \hbar \overleftarrow{D_{p_{2}^{\prime}}} \overrightarrow{D_{y^{\prime}}}\right) g
$$

where

$$
\begin{aligned}
& D_{x^{\prime}}=\partial_{x^{\prime}}+2 k t p_{2}^{\prime} \partial_{y^{\prime}}, \\
& D_{y^{\prime}}=\partial_{y^{\prime}}, \\
& D_{p_{1}^{\prime}}=\partial_{p_{1}^{\prime}}+\frac{1}{m_{1}} t \partial_{x^{\prime}}+\frac{k}{m_{1}} t^{2} p_{2}^{\prime} \partial_{y^{\prime}}, \\
& D_{p_{2}^{\prime}}=\partial_{p_{2}^{\prime}}-2 k t p_{2}^{\prime} \partial_{p_{1}^{\prime}}-\frac{k}{m_{1}} t^{2} p_{2}^{\prime} \partial_{x^{\prime}}+\left(\frac{1}{m_{2}} t+2 k t x^{\prime}-\frac{k}{m_{1}} t^{2} p_{1}^{\prime}-\frac{k^{2}}{m_{1}} t^{3} p_{2}^{\prime 2}\right) \partial_{y^{\prime}} .
\end{aligned}
$$

One can calculate that

$$
\begin{aligned}
\left(\hat{q}_{T}^{\prime}\right)^{1}= & x^{\prime}+\frac{1}{2} i \hbar \partial_{p_{1}^{\prime}}-\frac{1}{2}\left(\frac{i \hbar}{2}\right)^{2} \frac{k}{m_{1}} t^{2} \partial_{y^{\prime}}^{2} \\
\left(\hat{q}_{T}^{\prime}\right)^{2}= & y^{\prime}+\frac{1}{2} i \hbar \partial_{p_{2}^{\prime}}-\left(\frac{i \hbar}{2}\right)^{2} \frac{k^{2}}{m_{1}} t^{3} p_{2}^{\prime} \partial_{y^{\prime}}^{2}-\left(\frac{i \hbar}{2}\right)^{2} \frac{k}{m_{1}} t^{2} \partial_{x^{\prime}} \partial_{y^{\prime}} \\
& -\left(\frac{i \hbar}{2}\right)^{2} 2 k t \partial_{y^{\prime}} \partial_{p_{1}^{\prime}}+\frac{1}{3}\left(\frac{i \hbar}{2}\right)^{3} \frac{k^{2}}{m_{1}} t^{3} \partial_{y^{\prime}}^{3}, \\
\left(\hat{p}_{T}^{\prime}\right)_{1}= & p_{1}^{\prime}-\frac{1}{2} i \hbar \partial_{x^{\prime}}-\left(\frac{i \hbar}{2}\right)^{2} k t \partial_{y^{\prime}}^{2}, \\
\left(\hat{p}_{T}^{\prime}\right)_{2}= & p_{2}^{\prime}-\frac{1}{2} i \hbar \partial_{y^{\prime}},
\end{aligned}
$$


and that

$$
\left[\left(\hat{q}_{T}^{\prime}\right)^{i},\left(\hat{p}_{T}^{\prime}\right)_{j}\right]=i \hbar \delta_{j}^{i}
$$

The above equation shows that the transformation $T$ is a quantum canonical transformation. Now, using Theorem 3.1 it can be shown that the isomorphism $S_{T}$ is of the form

$$
S_{T}=\exp \left(\frac{1}{8} \hbar^{2} \frac{k}{m_{1}} t^{2} \partial_{x^{\prime}} \partial_{y^{\prime}}^{2}+\frac{1}{4} \hbar^{2} k t \partial_{p_{1}^{\prime}} \partial_{y^{\prime}}^{2}+\frac{1}{12} \hbar^{2} \frac{k^{2}}{m_{1}} t^{3} p_{2}^{\prime} \partial_{y^{\prime}}^{3}\right) .
$$

It can be also proved that $S_{T}$ is an isomorphism (unitary operator) of the Hilbert space $L^{2}\left(\mathbb{R}^{4}\right)$ onto itself.

It can be checked using formula (14) that the unitary operator $U_{T}$ associated to the transformation $T$ is of the form

$$
\begin{aligned}
\left(\hat{U}_{T} \varphi\right)\left(x^{\prime}, y^{\prime}\right)= & \frac{1}{(2 \pi \hbar)^{2}} \iint \varphi(x, y) \sqrt{\left|\operatorname{det}\left[\frac{\partial^{2} F}{\partial_{x^{i}} \partial_{p_{j}^{\prime}}}\left(x, y, p_{1}^{\prime}, p_{2}^{\prime}\right)\right]\right|} e^{\frac{i}{\hbar} F\left(x, y, p_{1}^{\prime}, p_{2}^{\prime}\right)} e^{-\frac{i}{\hbar}\left(x^{\prime} p_{1}^{\prime}+y^{\prime} p_{2}^{\prime}\right)} \mathrm{d} x \mathrm{~d} y \mathrm{~d} p_{1}^{\prime} \mathrm{d} p_{2}^{\prime} \\
= & \frac{1}{(2 \pi \hbar)^{2}} \iint \varphi(x, y) e^{\frac{i}{\hbar}\left(\left(x-x^{\prime}\right) p_{1}^{\prime}+\left(y-y^{\prime}\right) p_{2}^{\prime}+k t x p_{2}^{\prime 2}+\frac{1}{2 m_{1}} t p_{1}^{\prime 2}+\frac{1}{2 m_{2}} t p_{2}^{\prime 2}\right)} \\
& \times e^{\frac{i}{\hbar}\left(\frac{k}{2 m_{1}} t^{2} p_{1}^{\prime} p_{2}^{\prime 2}+\frac{k^{2}}{6 m_{1}} t^{3} p_{2}^{\prime 4}\right)} \mathrm{d} x \mathrm{~d} y \mathrm{~d} p_{1}^{\prime} \mathrm{d} p_{2}^{\prime}
\end{aligned}
$$

The transformation $T$ is an example of a quantum (and classical) trajectory on phase space of a two particle system which time evolution is described by the Hamiltonian [20]

$$
H\left(x, y, p_{1}, p_{2}\right)=\frac{p_{1}^{2}}{2 m_{1}}+\frac{p_{2}^{2}}{2 m_{2}}+k x p_{2}^{2} .
$$

The constants $m_{1}, m_{2}$ are then interpreted as masses of particles, $k$ is a coupling constant, and $t$ is an evolution parameter (time).

\section{Final remarks}

In the paper we presented consistent theory of canonical transformations of phase space coordinates in quantum mechanics. The starting point of this approach was considering phase space quantum mechanics and introducing there transformations of coordinates in a similar manner as in classical mechanics. Then we passed to ordinary description of quantum mechanics. Presented theory allows to solve the problem concerning the consistency of quantization mentioned in Introduction. Actually, one has to change the ordering of position and momentum operators in transformed observables from the symmetric to the $S_{T}$-ordering.

Returning to the example of the harmonic oscillator from Introduction we can now associate to the transformed Hamiltonian $H^{\prime}$ from (2) an appropriate operator. First, we need to calculate $S_{T}^{-1} H^{\prime}$, which using the form of the isomorphism $S_{T}$ calculated in the example from Subsection 5.3 gives

$$
\left(S_{T}^{-1} H^{\prime}\right)\left(x^{\prime}, p^{\prime}\right)=\left|x^{\prime}\right| p^{\prime 2}+\omega^{2}\left|x^{\prime}\right|+\frac{1}{16} \hbar^{2}\left|x^{\prime}\right|^{-1} .
$$

The $S_{T}$-ordered operator associated to $H^{\prime}$ is then equal

$$
H_{S_{T}}^{\prime}\left(\hat{q}^{\prime}, \hat{p}^{\prime}\right)=\left(S_{T}^{-1} H^{\prime}\right)_{M}\left(\hat{q}^{\prime}, \hat{p}^{\prime}\right)=\frac{1}{2}\left|\hat{q}^{\prime}\right| \hat{p}^{2}+\frac{1}{2} \hat{p}^{\prime 2}\left|\hat{q}^{\prime}\right|+\omega^{2}\left|\hat{q}^{\prime}\right|+\frac{1}{16} \hbar^{2}\left|\hat{q}^{\prime}\right|^{-1}
$$

This operator is indeed unitarily equivalent with its untransformed counterpart. The unitary operator $\hat{U}_{T}$ intertwining $H_{S_{T}}^{\prime}\left(\hat{q}^{\prime}, \hat{p}^{\prime}\right)$ and $H_{M}(\hat{q}, \hat{p})$ is of the form (20). To check that this is correct let us calculate the action of $H_{S_{T}}^{\prime}\left(\hat{q}^{\prime}, \hat{p}^{\prime}\right)$ on a ground state of the harmonic oscillator, $\varphi_{0}(x)=\left(\frac{\omega}{\pi \hbar}\right)^{1 / 4} \exp \left(-\frac{\omega x^{2}}{2 \hbar}\right)$, transformed to the new coordinate system by $\hat{U}_{T}$. One easily calculates that

$$
\left(\hat{U}_{T} \varphi_{0}\right)\left(x^{\prime}\right)=\left(\frac{\omega}{\pi \hbar}\right)^{1 / 4} \frac{1}{\sqrt{\left|2 x^{\prime}\right|}} \exp \left(-\frac{\omega\left|x^{\prime}\right|}{\hbar}\right)
$$


and that

$$
H_{S_{T}}^{\prime}\left(\hat{q}^{\prime}, \hat{p}^{\prime}\right) \hat{U}_{T} \varphi_{0}=\frac{1}{2} \hbar \omega \hat{U}_{T} \varphi_{0}
$$

which shows that $\hat{U}_{T} \varphi_{0}$ is an eigen-state of the transformed Hamiltonian of the oscillator, corresponding to an energy $\frac{1}{2} \hbar \omega$, as it should be.

The general theory of quantum canonical transformations developed in our paper suggests the proper approach for tackling the problem of general transformations of coordinates in quantum mechanics. Especially it provides a method of an investigation of non-canonical transformations, which are hard to introduce directly in ordinary quantum mechanics. In fact, the proper way of developing non-canonical transformation theory should look as follows. First we consider a transformation $T$ of coordinates in phase space quantum mechanics and find the form of the isomorphism $S_{T}$ related to $T$. Then with the help of $S_{T}$ we can pass to ordinary quantum mechanics. The problem which still needs to be solved is an introduction of a proper ordering of operators related to non-canonical coordinates $(\xi, \zeta)$, i.e., the definition of the operator function has to be extended so that the following formula would hold

$$
A \star_{T}^{\prime}=A_{S_{T}}\left(\xi \star_{T}^{\prime}, \zeta \star_{T}^{\prime}\right) .
$$

Only then the passage to ordinary quantum mechanics can be found.

In this paper we considered transformations of coordinates which were defined on almost the whole phase space. Problems arise when a transformation will be defined on some arbitrary open subset of phase space. We believe that in such case the space of states in ordinary quantum mechanics will be a Hilbert space of square integrable functions with respect to some measure dependent on the transformation. Moreover, the momentum operator would not be in the form $-i \hbar \partial_{x^{\prime}}$.

From the application point of view the problem which has to be solved is a systematic construction of isomorphisms $S_{T}$ and operators $\hat{U}_{T}$ for a general transformation $T$.

In conclusion, the possibility of introducing different coordinate systems in quantum mechanics is promising, however, there are still many problems which need to be solved. Furthermore, the geometric approach to coordinate transformations in phase space quantum mechanics, especially for quantum trajectories, gives a potential possibility of developing quantum theories similar to those found in classical theory, e.g., to develop a geometric theory of quantum integrable systems: quantum bi-Hamiltonian systems.

\section{References}

[1] M. Born, P. Jordan, W. Heisenberg, Z. Phys. 35 (1926) 557-615.

[2] P. Jordan, Z. Phys. 37 (1926) 383-386.

[3] P. Jordan, Z. Phys. 38 (1926) 513-517.

[4] F. London, Z. Phys. 37 (1926) 915-925.

[5] F. London, Z. Phys. 40 (1927) 193-210.

[6] P. A. M. Dirac, Proc. R. Soc. A 113 (1927) 621-641.

[7] P. A. M. Dirac, The Principles of Quantum Mechanics, Oxford University Press, New York, fourth edition, 1958.

[8] P. A. Mello, M. Moshinsky, J. Math. Phys. 16 (1975) 2017-2028.

[9] M. Moshinsky, T. H. Seligman, Ann. Phys. 114 (1978) 243-272.

[10] M. Moshinsky, T. H. Seligman, Ann. Phys. 120 (1979) 402-422.

[11] G. García-Calderón, M. Moshinsky, J. Phys. A 13 (1980) L185-L188.

[12] R. Dirl, P. Kasperkovitz, M. Moshinsky, J. Phys. A 21 (1988) 1835-1846.

[13] H. Lee, W. S. l'Yi, Phys. Rev. A 51 (1995) 982-988.

[14] J.-H. Kim, H.-W. Lee, Can. J. Phys. 77 (1999) 411-425.

[15] A. Anderson, Phys. Lett. B 319 (1993) 157-162.

[16] A. Anderson, Ann. Phys. 232 (1994) 292-331.

[17] J. Hietarinta, Phys. Rev. D 25 (1982) 2103-2117.

[18] T. Dereli, T. Hakioğlu, A. Teğmen, Int. J. Mod. Phys. A 24 (2009) 4573-4587.

[19] T. Curtright, D. B. Fairlie, C. Zachos, Phys. Rev. D 58 (1998) 25002-25015.

[20] N. C. Dias, J. N. Prata, J. Math. Phys. 45 (2004) 887-901.

[21] F. Bayen, M. Flato, C. Frønsdal, A. Lichnerowicz, D. Sternheimer, Ann. Phys. 111 (1978) 61-110.

[22] F. Bayen, M. Flato, C. Frønsdal, A. Lichnerowicz, D. Sternheimer, Ann. Phys. 111 (1978) 111-151.

[23] G. Dito, D. Sternheimer, in: G. Halbout (Ed.), Deformation quantization, volume 1 of IRMA lectures in mathematics and theoretical physics, Walter de Gruyter, Berlin, New York, 2002, pp. 9-54. 
[24] S. Gutt, in: G. Dito, D. Sternheimer (Eds.), Conférence Moshé Flato 1999: quantization, deformations, and symmetries, volume 21 of Mathematical physics studies, Kluwer Academic Publishers, Netherlands, 2000, pp. 217-254.

[25] A. Weinstein, in: Séminaire Bourbaki, volume 36, Association des Collaborateurs de Nicolas Bourbaki, 1993-1994, pp. 389-409.

[26] M. Błaszak, Z. Domański, Ann. Phys. 327 (2012) 167-211.

[27] F. Hansen, Rep. Math. Phys. 19 (1984) 361-381.

[28] J. B. Kammerer, J. Math. Phys. 27 (1986) 529-535.

[29] J. M. Maillard, J. Geom. Phys. 3 (1986) 231-261.

[30] J. M. Gracia-Bondía, J. C. Várilly, J. Math. Phys. 29 (1988) 869-879.

[31] M. Bordemann, S. Waldmann, Commun. Math. Phys. 195 (1998) 549-583.

[32] S. Waldmann, Rev. Math. Phys. 17 (2005) 15-75.

[33] N. C. Dias, J. N. Prata, Ann. Phys. 313 (2004) 110-146.

[34] M. Błaszak, Z. Domański, Quantum trajectories, 2012. Eprint arXiv:1208.0720v1 [math-ph]. 